Check for updates

Cite this: Mater. Adv., 2021, 2, 7118

Received 31st March 2021 Accepted 6th September 2021 DOI: 10.1039/d1ma00290b

rsc.li/materials-advances

\section{Mechanisms for doped PEDOT:PSS electrical conductivity improvement}

\author{
Nur'Aishah Ahmad Shahrim, (D) ${ }^{a}$ Zuraida Ahmad, ${ }^{\star a}$ Amelia Wong Azman, ${ }^{b}$ \\ Yose Fachmi Buys ${ }^{\mathrm{C}}$ and Norshahida Sarifuddin ${ }^{\mathrm{a}}$
}

\begin{abstract}
Due to their good electrical conductivity and versatility, conductive polymers (CPs), in particular, poly(3,4-ethylene dioxythiophene) (PEDOT):poly(styrene sulphonate) (PSS), have recently attracted considerable research interest in bioelectronics applications. This study provides insight into the mechanisms in PEDOT:PSS for increasing electrical conductivity. As such, the preparation of doped PEDOT:PSS using distinctive approaches, such as undergoing treatment and using secondary dopants is focused primarily on improving its electrical efficiency. It also systematically addresses various primary parameters that have significant effects on its conductivity. We present the potential of doped PEDOT:PSS for many promising applications in fields such as bioelectronics, through an in-depth analysis of the most remarkable studies recorded by various research groups over the past decade. Therefore, this review is expected to be significantly helpful in promoting further studies, as well as paving the way for increased qualification and productivity for future revolutions of organic CP materials.
\end{abstract}

\section{Introduction}

Conductive polymers (CPs) are well-known as smart materials that combine the unique properties of metals such as charge transfer and electrical and optical properties; traditional polymers, in particular, have versatility in processing and the ease of synthesis. ${ }^{1,2}$ This is because of their abilities to deliver electrical, electrochemical, and electromechanical stimulations directly to the target. The discovery of the conductive polymers was made in 1977 through the breakthrough of the high

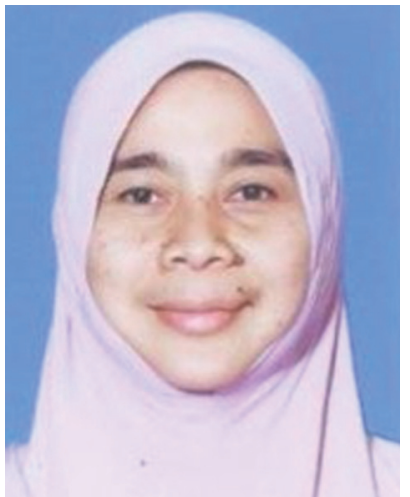

Zuraida Ahmad
Zuraida Ahmad received her PhD Nur'Aishah Ahmad Shahrim received her BS Hons. in Materials Engineering (2017) and MSc. Materials Engineering (2019) from International Islamic University Malaysia (IIUM). She is currently pursuing her PhD in Materials Engineering at the same university. Her research focuses on the preparation and characterization of conductive polymers as bioelectronics for biomedical applications. Also, her research interests lie in the biopolymers for packaging industrial applications. in Engineering (Materials) from International Islamic University Malaysia (IIUM). She joined Standard and Industrial Research Institute of Malaysia (SIRIM) Berhad in 1993 and has successfully lead projects related to development of composites for industrial applications. Later in 1999, she joined IIUM as lecturer until present and holds an Associate Professor position. Her research interests lie in bio-

materials for biomedical applications. She also has research interests in polymers, ceramics, and composites for packaging industrial applications. 
conductivity of polyacetylene-doped iodine as a p-type dopant (oxidizing agent). Subsequently, in 1978, polyacetylene mixed with an n-type dopant (reducing agent) depicted a conductivity effect. These early works on polyacetylene have stimulated research interest in utilizing conductive polymers for enduser applications. Interestingly, these CPs possess excellent electrical and electronic properties, while allowing excellent control of the electrical stimulus due to the presence of conjugated backbone $\pi$-electrons. ${ }^{3,4}$ In addition, the great benefits of CPs are that their chemical, electrical, and physical properties can be adapted to their particular application needs by undergoing doping or reversible doping processes. Specifically, CPs can be fully applied in bioelectronics, such as tissue engineering or regenerative medicine, which aims to substitute or recover malfunctioning or failing tissue by merging bioactive molecules, cells, and scaffolds. ${ }^{5,6}$ It is noted that there is information on CPs for bioelectronics such as biosensors, neural probes, neural prostheses, and controlled release systems. ${ }^{7,8}$

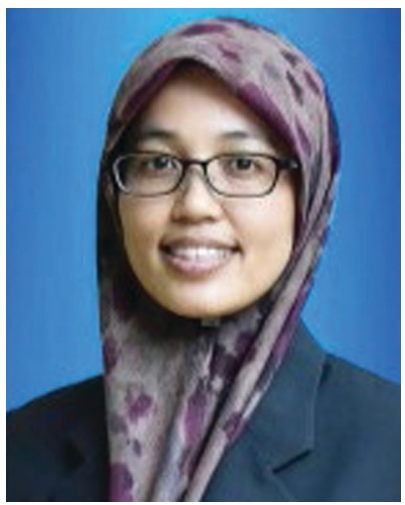

Amelia Wong Azman

Amelia Wong Azman received her $P h D$ in Electronics from University of Queensland, Australia in 2011. She joined National ICT Australia (NICTA) and has contributed to the research on smart surveillances. She is currently holding an Associate Professor at International Islamic University Malaysia (IIUM). Her research interests lie in rehabilitation engineering in reconfigurable architecture system design. She also has research interests in digital and analogue electronics, as well as Information, Computer and Communications Technology (ICT).

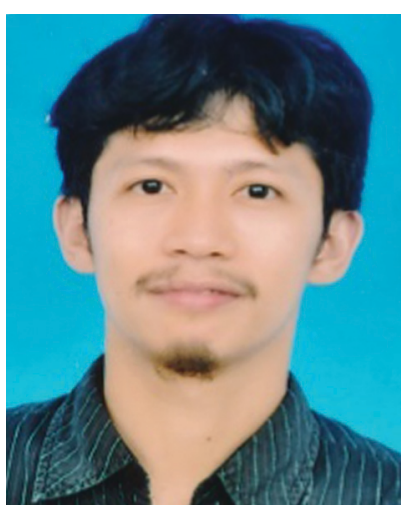

Yose Fachmi Buys
Yose Fachmi Buys received his PhD in Organic and Polymeric Materials from Tokyo Institute of Technology, Japan. He worked as a researcher at Texchem Polymers in 2010 and later joined International Islamic University Malaysia (IIUM) as an Assistant Professor in 2013 and a senior lecturer at University of Malaya in 2017. Currently, he is assigned as lecturer at Universitas Pertamina, Indonesia. His research interests lie in conduc-

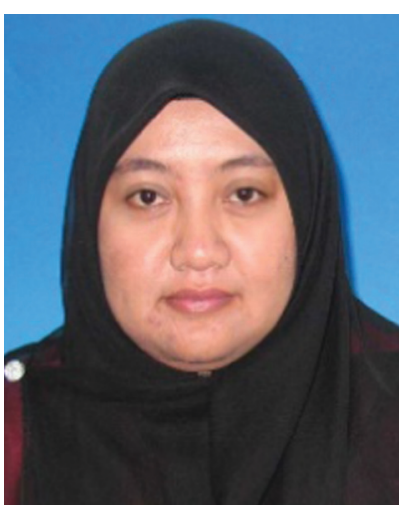

Norshahida Sarifuddin
Norshahida Sarifuddin received her PhD in Biopolymer Engineering from University of Science Malaysia (USM). She worked at CTRM Aero Composites as a materials engineer and later joined International Islamic University Malaysia as an Assistant Professor in 2015 until present. Her research interests lie in biopolymers and biocomposites for packaging industrial applications. Her research interests also lie in hybrid composites for its tribological behavior.

tive polymer composites. He also has research interests in thermoplastic, polymer compounds, composite materials, and polymers. 
Table 1 Properties of commercialized PEDOT:PSS products

\begin{tabular}{|c|c|c|c|c|c|}
\hline $\begin{array}{l}\text { PEDOT:PSS } \\
\text { commercial name }\end{array}$ & $\begin{array}{l}\text { Conductivity } \\
\left(\mathrm{S} \mathrm{cm}^{-1}\right)\end{array}$ & $\begin{array}{l}\text { PEDOT: PSS ratio } \\
\text { (by weight) }\end{array}$ & $\begin{array}{l}\text { Solid } \\
\text { content }(\%)\end{array}$ & Commercial use & Ref. \\
\hline Clevios PH 500 & $0.2-1$ & $1: 2.5$ & $1.0-1.4$ & Conductive and stretchable electrode for electronic devices & $16-21$ \\
\hline Clevios PH1000 & $0.2-1$ & $1: 2.5$ & $1.0-1.3$ & Conductive electrode and stretchable for electronic devices & $22-43$ \\
\hline Clevios PVP AI 4083 & $10^{-3}-10^{-4}$ & $1: 6$ & $1.3-1.7$ & $\begin{array}{l}\text { Hole transport/injection layer of organic electronics, } \\
\text { inkjet printing }\end{array}$ & 34 and $48-54$ \\
\hline Clevios PVP CH 8000 & $10^{-5}-10^{-6}$ & $1: 20$ & $2.5-3.0$ & Hole transport/injection layer of organic electronics & 55 \\
\hline
\end{tabular}

electrical conductivity of PEDOT:PSS is lower than that of other conductive polymers or metal oxides, which is less than $1 \mathrm{~S} \mathrm{~cm}^{-1}$, making PEDOT:PSS a standalone electrode that is unsuitable for bioelectronics application. ${ }^{57,58}$ Fortunately, it is possible to boost the conductivity by using secondary dopants, in particular, by introducing organic solvents such as ethylene glycol (EG) and dimethyl sulfoxide (DMSO), ${ }^{59-66}$ or by introducing poly(alcohols) such as sorbitol and glycerol, ${ }^{67-71}$ or by adding surfactants such as sodium dodecyl sulfate (SDS), hexadecyltrimethylammonium bromide (CTAB), and poly(oxyethylene) phenyl ether, ${ }^{72,73}$ or by treating acids such as phosphoric acid $\left(\mathrm{H}_{3} \mathrm{PO}_{4}\right)$, sulfuric acid $\left(\mathrm{H}_{2} \mathrm{SO}_{4}\right)$, and sulfonic acid. ${ }^{74-76}$ Based on the literature, the transparent and highly conductive PEDOT:PSS films have been studied extensively, particularly for replacing indium tin oxide (ITO) in photovoltaics (OPV), ${ }^{77-80}$ solar cells, ${ }^{81-83}$ OLED devices, ${ }^{84}$ and touch screens.$^{85}$ Furthermore, PEDOT:PSS has currently been documented in bioelectronic applications, such as stretchable and wearable electronics. ${ }^{86,87}$ Hence, due to its significant advantages over other CPs, PEDOT:PSS has great potential as a transparent electrode substrate of the next generation. Furthermore, PEDOT:PSS is easy to use, highly stable in environmental conditions, has strong clarity in the field of visible light and various conductivity applications using secondary doping products that are appropriate for different applications.

Although such high conductivities of PEDOT:PSS are achieved, the fundamental mechanisms for the observed enhancement of conductivity are often debated and considered controversial $^{88}$ Therefore, further efforts are required to clarify the origins of this improved conductivity for PEDOT:PSS. In addition, the statistics found in the Web of Science indicate that literature related to PEDOT:PSS conductivity has been published in over 100 distinct journals with over 200 research papers in the last decade, causing considerable difficulty in further study. A logical overview of randomly distributed literature concerning the PEDOT:PSS conductivity enhancement approaches and processes is a critical requirement. This would be an important guide for PEDOT:PSS research, manufacture, and implementation in the near future.

Therefore, this review primarily emphasizes the mechanism for enhancing PEDOT:PSS conductivity through both physical and chemical approaches. It also addresses the use of highly conductive PEDOT:PSS films and hydrogels in bioelectronics, as well as the prospects for future research efforts. Given the vast number of new possibilities that PEDOT:PSS could bring, it is hoped that the review will provide insight for revolutionizing the engineering world, specifically the biomedical field.

\section{Approaches to tailoring PEDOT:PSS conductivity}

PEDOT:PSS has a complex structure due to the combination of two polyelectrolytes. The chemical structures of PEDOT:PSS are shown in Fig. 1a and b to counterbalance the charge of the carriers in conjugation with the conjugated poly(3,4-ethylene dioxythiophene) (PEDOT) and the poly(styrene sulfonate) (PSS). Specifically, based on Fig. 1b, the -OH groups in the PSS structure disassociated to give $\mathrm{H}^{+}$in water, then the $\mathrm{H}^{+}$deliberately targeted the double bond of the PEDOT's thiophene, which led to the formation of hydrogen bonding. At the same time, the electrons of the $\mathrm{C}-\mathrm{O}$ mutual electron pairs were attracted by $\mathrm{O}$ due to their higher electronegativity. This phenomenon was preceded by the fact that the $\mathrm{C}$ of the $\mathrm{C}-\mathrm{O}$ bond had become a positive charge. The ionic bond was formed between the $\mathrm{C}^{+}$and the $\mathrm{O}^{-}$of the PSS structures. ${ }^{64} \mathrm{In}$ addition, the chemical structure of PEDOT:PSS can be compared with the morphological model shown in Fig. 1c, which demonstrates its formation of colloidal gel particles when dispersed in water, and the resulting films are microstructured with PEDOT: PSS-rich (blue) and PSS-rich (grey) domains. Small segments of PEDOT are in close contact with the PSS bundles in the form of a solution. This observation results in the formation of a colloid of gel particles in water. This is due to the difference in molecular weight, resulting in a large density of the two components. The average molecular weight of PEDOT is approximately $1000 \mathrm{~g} \mathrm{~mol}^{-1}$, while the molecular weight of PSS is approximately $400000 \mathrm{~g} \mathrm{~mol}^{-1} \cdot{ }^{70}$ As such, the density of PEDOT is the highest in the center of the particles, while the density of the hydrophilic PSS is the highest in the periphery. As such, the deposition and drying of PEDOT:PSS led to the formation of a pancake-like morphology of grains with a PEDOT-rich center and a PSS-rich shell. The PEDOT:PSS morphology thus plays a major role in determining the PEDOT:PSS conductivity mechanism. This covers the effects of the processing method and any other additives (commonly known as "secondary dopants") incorporated into the recipe to enhance the conductivity for the intended applications.

The conductivity of the film produced from the aqueous PEDOT:PSS suspension depends largely on the ratio of the two components. It is noteworthy that even though the PEDOT:PSS film was prepared with nearly the same conductivity as the as-prepared PEDOT:PSS aqueous solutions (Table 1), typically within 0.1-1 S cm${ }^{-1}$, the increase in the PEDOT:PSS films conductivity is strongly influenced by the grade. ${ }^{22}$ For example, 


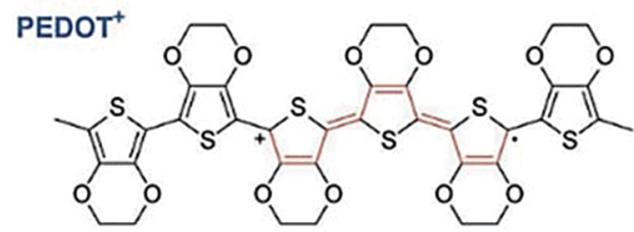

PSS $^{-}$

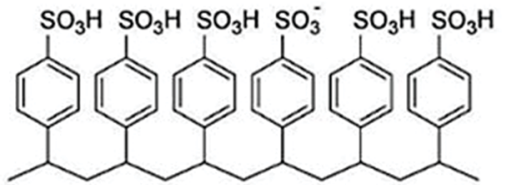

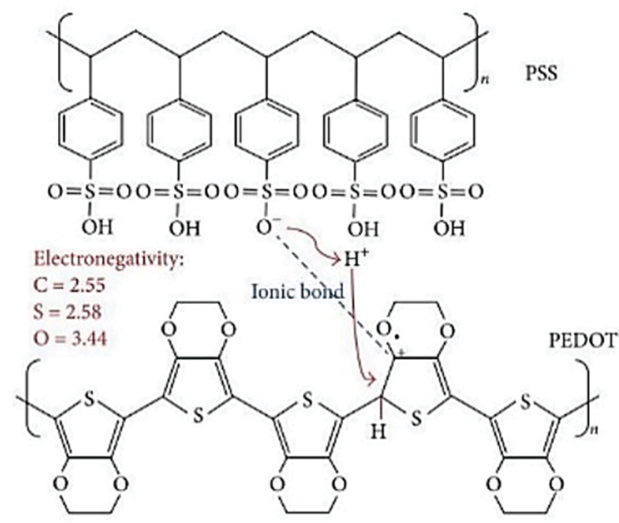

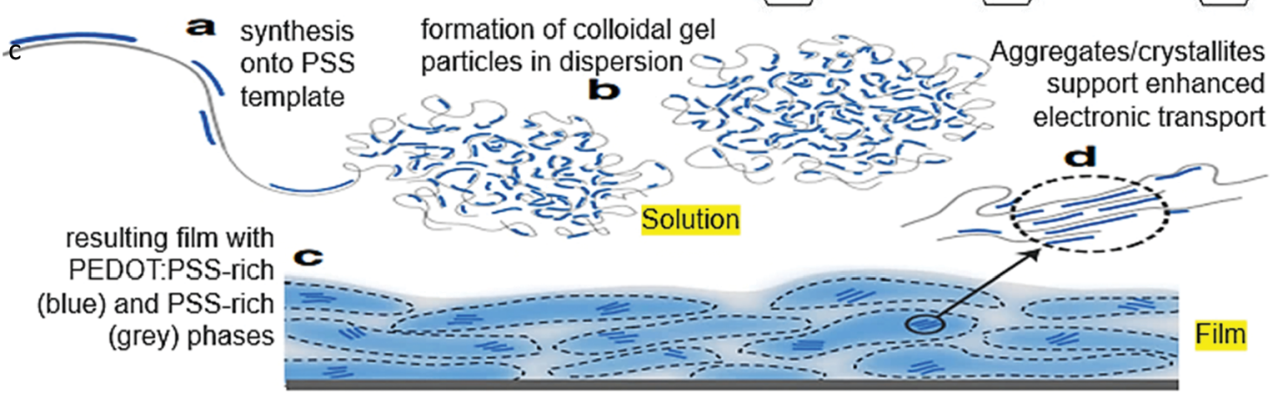

Fig. 1 The (a) chemical structure, (b) reaction, and (c) morphological scheme of PEDOT and PSS. Reproduced with permission, ${ }^{18}$ Copyright $\mathbb{C} 2013$, Hindawi. ${ }^{93}$ Copyright $($ 2016, Springer Nature.

the Clevios P film conductivity may be up to $200 \mathrm{~S} \mathrm{~cm}^{-1}$, while the Clevios PH1000 film conductivity may be up to 600$700 \mathrm{~S} \mathrm{~cm}^{-1}$ after DMSO or EG treatment. ${ }^{89}$ In addition, conductivities above $1000 \mathrm{~S} \mathrm{~cm}^{-1}$ have also been reported for treated Clevios PH1000 films. PEDOT:PSS films from Clevios PH1000 aqueous solution added with EG were doubly treated in the EG bath. As a result, the conductivity of PEDOT:PSS films improved to $1418 \mathrm{~S} \mathrm{~cm}^{-1} \cdot{ }^{77}$ Clevios PH1000 achieved $1164 \mathrm{~S} \mathrm{~cm}^{-1}$ film conductivity through treatment with hexafluoroacetone trihydrate (HFA). ${ }^{22}$ Further improvement on conductivity to over $3000 \mathrm{~S} \mathrm{~cm}^{-1}$ was achieved by treatment with sulfuric acid $\left(\mathrm{H}_{2} \mathrm{SO}_{4}\right)$ for Clevios $\mathrm{PH} 1000 .{ }^{90,91}$ This conductivity on plastic substrates is much higher than that of indium tin oxide (ITO) and equivalent to ITO on the glass substratum. Nonetheless, the issues of scarcity, which causes the price of indium to skyrocket, and high mechanical frailty, are important to point out. Such concerns are a problem for the long-term availability of ITO and could cause ITO to be inadequate for versatile electronic devices of the next generation. PEDOT:PSS displays great potential as an alternative to ITO as the transparent electrode for optoelectronic equipment.

Therefore, over the last decades, a lot has been done to enhance the conductivity of PEDOT:PSS. The lack of electrical conductivity is due to the insulating PSS shell that restricts the transmission of charge across the grains, even though the core is rich in conductive PEDOT. Therefore, various approaches have been conveyed to dramatically enhance PEDOT:PSS's conductivity by tailoring this electrical property. These approaches include (1) solvent doping treatment, (2) solvent doping combined with post-treatment, (3) ionic liquid treatment, (4) acid treatment, and (5) the addition of metal nanoparticles. These approaches will be comprehensively described and comparatively studied.

\subsection{Treatment by solvent doping}

The chemical procedure known as solvent doping treatment provides a means for improving PEDOT:PSS's conductivity. Here, a polar organic compound with a high boiling point is added to the PEDOT:PSS solution or used to treat the PEDOT: PSS film. Table 2 summarizes the key results of several conductivity-enhancement studies for doped PEDOT:PSS with organic solvents such as ethylene glycol (EG), polyethylene glycol (PEG), diethylene glycol, dimethyl sulfoxide (DMSO), dimethyl sulfate, tetrahydrofuran (THF), $N, N$-dimethyl formamide (DMF), sorbitol, glycerol, meso-erythritol, xylitol, and methoxy ethanol. Other organic solvents can be classified into the $\mathrm{OH}$ group, such as ethanol $(\mathrm{EtOH})$ and methanol $(\mathrm{MeOH})$, while acetone can be classified into the non-OH group. A major improvement of the conductivity ranging from $0.5 \mathrm{~S} \mathrm{~cm}^{-1}$ to $1233 \mathrm{~S} \mathrm{~cm}^{-1}$ can be achieved depending on the process and the solvent. Usually, the high boiling solvent is applied before the deposition to the PEDOT:PSS dispersion, and thus this approach is called the solvent additive method. Therefore, based on the previous research done to improve the conductivity of PEDOT:PSS by the solvent additive method, it can be said that this type of approach is simple, versatile, and easy to process. The process is direct and the conductivity of the doped PEDOT:PSS can be characterized without any difficulty. This approach is more convenient to researchers for evaluating the 
Table 2 Applications of solvents as the secondary dopant to enhance the conductivity of PEDOT:PSS as reported in previous studies

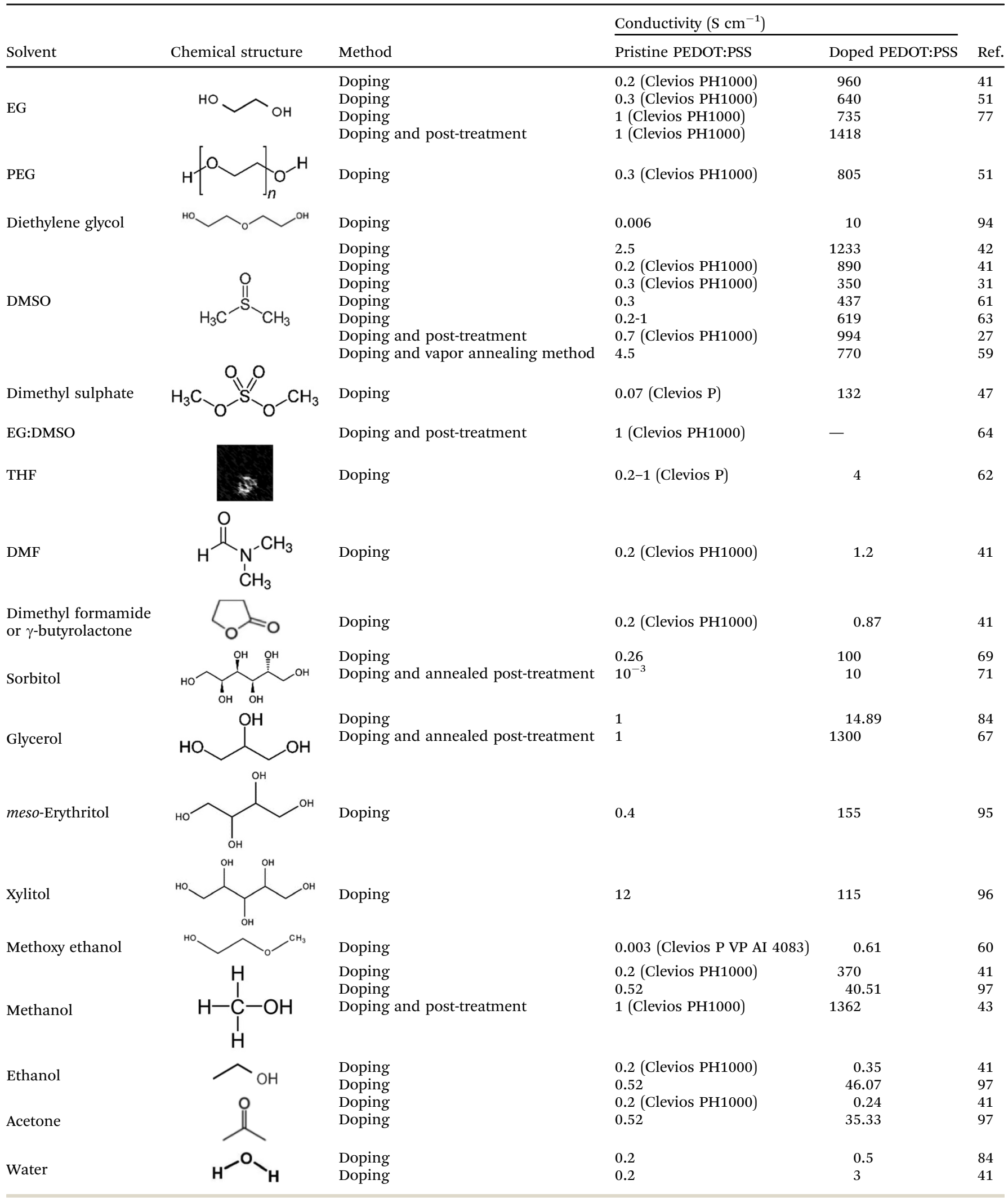

electrical performance of the PEDOT:PSS for specific applications such as sensors and solar cells. It is also noted that the utilization of different grades of PEDOT:PSS would result in different conductivities after the addition of the solvent. For 
example, the addition of ethylene glycol to the PEDOT:PSS has been reported in many studies. ${ }^{25,92}$ Even though the researchers claimed to utilize PEDOT:PSS grade Clevios PH1000, the initial conductivity differed: $0.2 \mathrm{~S} \mathrm{~cm}^{-1}, 0.3 \mathrm{~S} \mathrm{~cm}^{-1}$, and $1 \mathrm{~S} \mathrm{~cm}^{-1}$. Yet, the improvements in the PEDOT:PSS conductivity by solvent doping treatment were similar, namely, two orders of magnitude. Other types of solvent also improved the conductivity of PEDOT:PSS by one to two orders of magnitudes. The different types of solvent give various conductivities for PEDOT:PSS because these solvents have their own dielectric constants at room temperature due to the dipole moment between the solvent molecules. ${ }^{62}$ The higher the dielectric constant of a solvent like DMSO, the stronger the screening effect between counterions and charge carriers, thereby minimizing the Coulomb interaction between the positively charged PEDOT and the negatively charged PSS. Mechanisms for the PEDOT:PSS conductivity improvement will be discussed in a subsequent section.

However, the solvent additive approach to enhancing the electrical conductivity of PEDOT:PSS has the disadvantage of insufficient conductivity being achieved for certain applications such as organic solar cells. For instance, PEDOT:PSS with the addition of ethylene glycol achieved a conductivity of $735 \mathrm{~S} \mathrm{~cm}^{-1}$, while a solvent post-treatment method remarkably increased the conductivity to $1418 \mathrm{~S} \mathrm{~cm}^{-1} .^{77}$ The mechanism for this conductivity improvement will be discussed in the next section (Section 2.2). As such, it is suggested that an additional process is performed to improve the PEDOT:PSS conductivity after the addition of the solvent.

The post-treatment method would enhance the conductivity of the resulting PEDOT:PSS film, in addition to adding solvents to the PEDOT:PSS solutions. In general, thermal annealing treatment has led to improvements in the conductivity and stability due to decreased water absorption by denser PEDOT chains packing. It has been confirmed that the PEDOT:PSS aqueous solution achieved an increase in conductivity when sorbitol was applied, and the resulting film was then baked at a high temperature of more than $100{ }^{\circ} \mathrm{C} .{ }^{68}$ The interchain interactions in the PEDOT:PSS film decreased at high temperature as the additive acted as a plasticizer to help re-orient the PEDOT chains. On the other hand, methanol caused a screening effect between positively charged PEDOT chains and negatively charged PSS chains. This behavior suggests a reduction in the coulombic interaction between them, as is the case with high boiling points for secondary dopants. This will lead to the separation of phases on a nanometer scale, as shown by the segregation of the excess PSS. ${ }^{43}$ Also, the highly polar hydrophilic methanol can easily dissolve the PSS-separated hydrophilic phase and facilitate removal from the film. This behavior would promote the reorientation of the PEDOT polymer chains from coiled to linear or extended coil form, enabling the conductive polymers to be more interchained. As a result, there will be a lowering of the energy barrier for inter-chain and interdomain charge hopping and a smoother transfer of charges between PEDOT chains. The phase-separated and re-oriented PEDOT chains will make it easier for the charges to hop, and a massively enhanced conductivity will eventually be achieved.
It can be concluded that the post-treatment method, whether it is carried out using high temperature or solvent addition after the PEDOT:PSS film was formed (also known as the post-deposition method), does play a vital role in the arrangement of the PEDOT and PSS molecules within the chains. Based on previous studies, it is proposed that the doped PEDOT:PSS film undergoes a thermal annealing process for a better conductivity improvement of up to three orders of magnitude.

\subsection{Treatment with ionic liquids}

Apart from solvent additive and post-treatment method to tackle the low conductivity of PEDOT:PSS, ionic liquids could also be a potential solution. Organic and inorganic salts identified as ionic liquids (ILs) have distinctive attributes like good chemical stability, low ignition, insignificant vapor pressure, and non-volatility. This kind of dopant is broadly used in many areas of technology such as optoelectronics. The addition of various ILs in the Baytron P PEDOT:PSS has demonstrated significant conductivity enhancement. ${ }^{58}$ In particular, numerous imidazoles have been synthesized: 1-butyl-1-methylpyrrolidium chloride (43 $\mathrm{S} \mathrm{cm}^{-1}$ ), 1-benzyl-3-methylimidazolium chloride (49 $\mathrm{S} \mathrm{cm}^{-1}$ ), 1-ethyl-3-methylimidazolium chloride (55 $\mathrm{S} \mathrm{cm}^{-1}$ ), 1-butyl-3-methylimidazolium bromide $\left(118 \mathrm{~S} \mathrm{~cm}^{-1}\right)$, and 1-butyl-3-methylimidazolium tetrafluoroborate $\left(136 \mathrm{~S} \mathrm{~cm}^{-1}\right)$. One can conclude that ILs demonstrate excellent performance as accompaniments in PEDOT:PSS films for enhancing the conductivity. The conductivity enhancement of PEDOT:PSS films by adding 2-methylimidazole, a derivative of imidazole was reported. ${ }^{35}$ The incorporation of synthesized 2-methylimidazolium hydrogen sulfate, 2-methylimidazolium bromide, and 2-methylimidazolium chloride in the PEDOT:PSS has been proven to improve conductivity. ${ }^{99}$ It was assumed that the mechanism for enhancing PEDOT:PSS conductivity via ionic liquids resulted in the phase segregation of PSSH chains from PEDOT: PSS and the conformational change of PEDOT chains. PEDOT: PSS composition and structure can also be altered by acidic ionic liquids. To protect the coulombic attraction between PEDOT and PSS, the PSS chains can be separated by the ionic liquidmediated phases. Any of those PSS chains can be removed by washing with water from polymer solutions. This act precedes the improvement of conductivity for PEDOT:PSS by reducing the less conductive shell, which is the PSS. At the same time, AFM showed that doped PEDOT:PSS has a clearer and flatter film, showing that the insulating PSS was washed from the PEDOT: PSS surfaces, leaving a higher conductive layer with a large amount of PEDOT chains on the surface (Fig. 2). The increase in conductivity is also associated with a screening effect between the positively charged PEDOT chains and the negatively charged PSS chains, while weakening their coulombic interaction.

The utilization of ILs as a secondary dopant for PEDOT:PSS is very limited. Only a few studies have focused on the effect of incorporating ILs on the electrical conductivity of PEDOT:PSS films, ${ }^{58,88,95,98-100}$ but it could be said that the results obtained from these studies are promising. However, it is not recommended that ILs be incorporated with PEDOT:PSS for biological applications. A more concise understanding of these ILs on the 
(a)

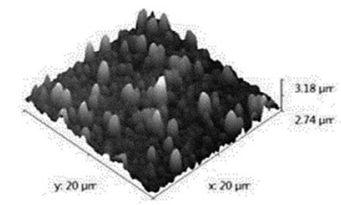

(b)

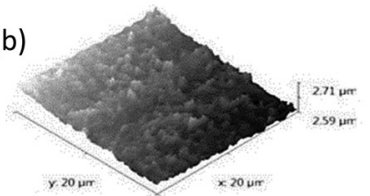

(c)

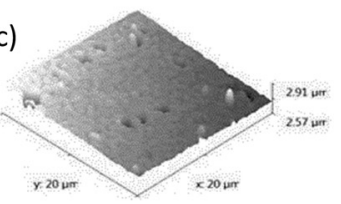

Fig. 2 AFM images of (a) untreated PEDOT:PSS and that treated with (b) 2-methylimidazolium chloride, and (c) 2-methylimidazolium hydrogen sulfate films. Reproduced with permission, ${ }^{98}$ Copyright $(\mathbb{C} 2015$, Taylor $\&$ Francis.

conductivity improvement of PEDOT:PSS is required for further research.

\subsection{Treatment with acid}

Acid treatment usually consists of soaking the as-cast PEDOT: PSS films in strong acid to enhance the electrical conductivity. Alternatively, for the PEDOT:PSS films cast at high temperatures of around $120{ }^{\circ} \mathrm{C}$, aqueous dilute acid solution is employed. Some studies have revealed the efficacy of the strong acid soaking treatment to boost the conductivity of PEDOT:PSS films by three orders of magnitude, or by up to $3000 \mathrm{~S} \mathrm{~cm}^{-1}$. The post-treatment approach is generally a more widely used process for treating acids. Sulfuric acid $\left(\mathrm{H}_{2} \mathrm{SO}_{4}\right)$ and hydrochloric acid $(\mathrm{HCl})$ are also used to effectively enhance PEDOT:PSS conductivity. Findings regarding the impact of acid treatment on the electrical conductivity of PEDOT:PSS are summarized in Table 3.

The enhancement of the PEDOT:PSS conductivity was confirmed when treated with $1 \mathrm{M}$ and $100 \% \mathrm{H}_{2} \mathrm{SO}_{4} ;{ }^{74,75}$ the conductivity was enhanced by up to three orders of magnitude. Further enhancement of PEDOT:PSS conductivity up to four orders of magnitude has been achieved by the incorporation of various sulfonic acids into PEDOT:PSS. ${ }^{20}$ The acid-like chloroplatinic acid $\left(\mathrm{H}_{2} \mathrm{PtCl}_{6}\right)$ has a good predisposition to establish $\mathrm{PtCl}_{2} ; \mathrm{Pt}^{4+}$ ions are released to $\mathrm{Pt}^{2+}$, and at the same time, PEDOT chains are oxidized to create more charge carriers. On the other hand, in pristine films, PEDOT chains are wrapped by Coulomb forces by PSS, and coil conformations and resonant benzenoid structures are introduced. Charges are located in such structures in the PEDOT chains where the contact points for two PEDOT chains are restricted, and there is an extreme energy hopping barrier. Also, $\mathrm{H}_{2} \mathrm{PtCl}_{6}$ will dissociate into anions and $\mathrm{H}^{+}$in PEDOT:PSS aqueous solution. The $\mathrm{H}^{+}$will bind directly to the $\mathrm{SO}^{3-}$ groups in PSS, thereby reducing their negative charges and the coulombic interactions between PEDOT and PSS, eventually resulting in the phase separation of the two components. This separation step allows PEDOT the opportunity to attain a linear chain conformation and quinoid resonant structure, which tends to be excellent at providing longer paths and more points of contact between PEDOT chains for charge transport. Therefore, the carrier efficiency is enhanced by more than two orders of magnitude. The conductivity of PEDOT:PSS is also hugely improved as both the carrier concentration and mobility are increased.

Recently, hydrobromic acid ( $\mathrm{HBr}$ ) treatment was used to achieve PEDOT:PSS film with high conductivity. ${ }^{99}$ The studies looked at the effect of post-treatment with acid deposited on the surface of the PEDOT:PSS thin film. The mechanism of the conductivity enhancement for the pristine PEDOT:PSS after $\mathrm{HBr}$ post-treatment is due to the change in the structure conformation, where a one-dimensional fibril-like structure was observed under an atomic force microscope (AFM). This is because the PSS chain was separated from the coiled structure of chains that were joined together by large PEDOT:PSS core-shell particles. The PSS chain separation was also confirmed by X-ray photoelectron spectroscopy (XPS), which showed that the intensity ratio of the peaks corresponding to sulfur atoms in PEDOT to PSS increased after treating PEDOT:PSS with HBr. Further progress in the high conductivity resulting from the $\mathrm{HBr}$ treatment needs to be investigated for appropriate applications.

The preparation of PEDOT:PSS films by acid treatment is more effective than the solvent additive method according to the factor of conductivity enhancement reported by previous research. However, acid treatment would not be suitable for certain applications such as bioelectronics for biomedical applications. It is not recommended that acids be utilized to improve the conductivity of PEDOT:PSS for biological application due to the use of the endproduct in direct contact with a person's skin. Acids are well known as hazardous chemicals and are thus unsafe for human skin.

\subsection{Metal nanoparticles}

Another approach that has attracted much effort from researchers is the incorporation of metal nanoparticles into PEDOT:PSS. The hybridization between the conducting polymers and metallic nanoparticles is expected to enhance the electrical conductivity for appropriate applications such as in the biomedical field. Table 4 lists the metal nanoparticles used for incorporation into PEDOT:PSS, as reported by numerous studies.

Silver nanoparticles were synthesized before doping with PEDOT:PSS. This is because silver has the highest conductivity among other metals, according to the periodic table. Silver nanoparticles have attracted significant interest for synthesis and doping into PEDOT:PSS for conductivity improvement. Silver also possesses antimicrobial properties, hence it is suitable for biomedical applications. Specifically, the synthesis of silver nanoparticles was carried out in different manners. Interestingly, the most common method was the in situ method through the chemical reduction of silver nitrate $\left(\mathrm{AgNO}_{3}\right)$ by sodium borohydride $\left(\mathrm{NaBH}_{4}\right)$. The reaction between silver nitrate and sodium borohydride is given as follows: ${ }^{104}$

$$
\mathrm{AgNO}_{3}+\mathrm{NaBH}_{4} \rightarrow \mathrm{Ag}+1 / 2 \mathrm{H}_{2}+1 / 2 \mathrm{~B}_{2} \mathrm{H}_{6}+\mathrm{NaNO}_{3}
$$

The synthesis of silver nanoparticles is predicted to occur from $\mathrm{Ag}^{+}$in the presence of PEDOT:PSS, which is reduced by $\mathrm{NaBH}_{4}$ to $\mathrm{Ag}_{0}$. The silver atoms expand as clusters to form 
Table 3 Acid modifications of the conductivity of PEDOT:PSS reported in the literature

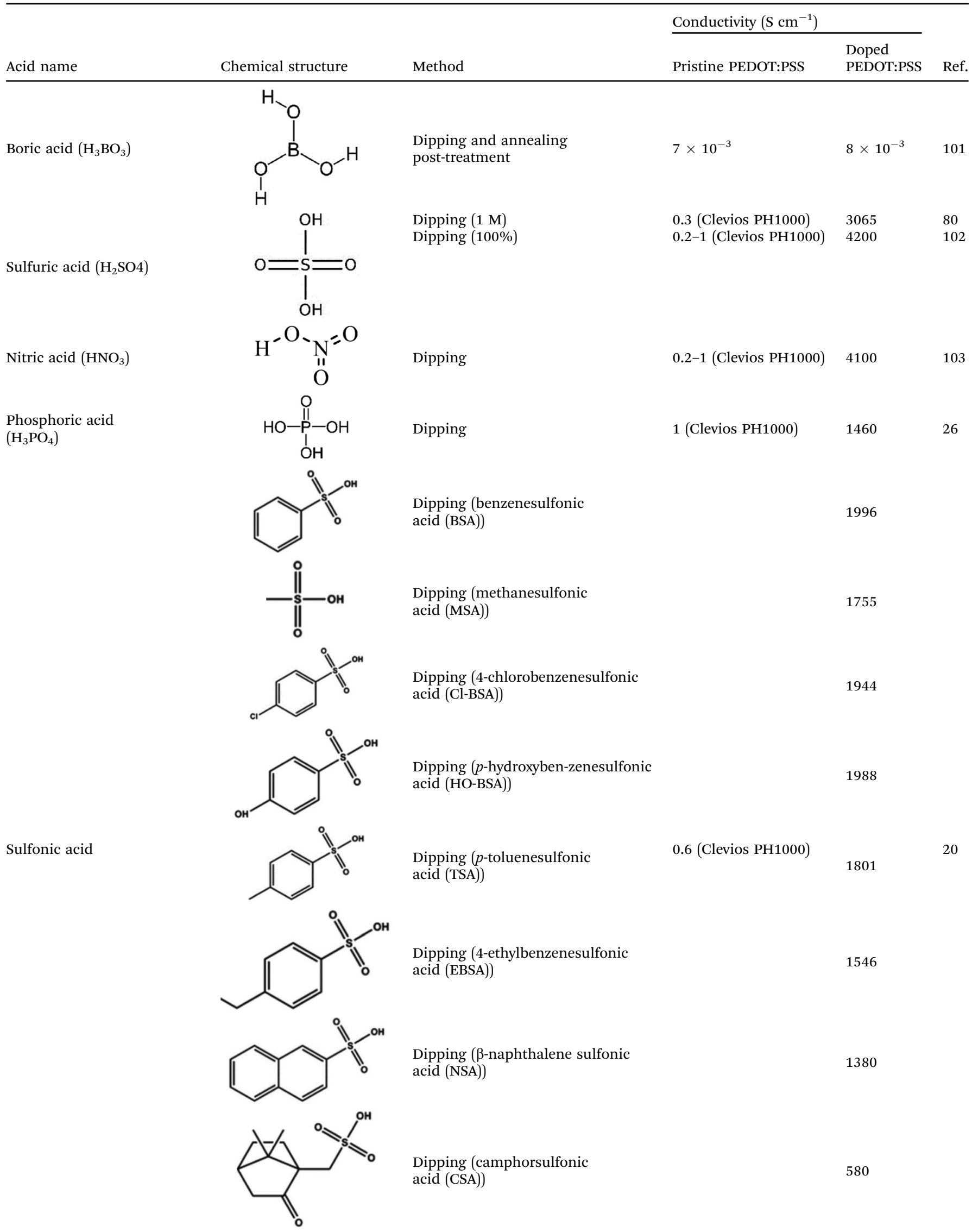


Table 3 (continued)

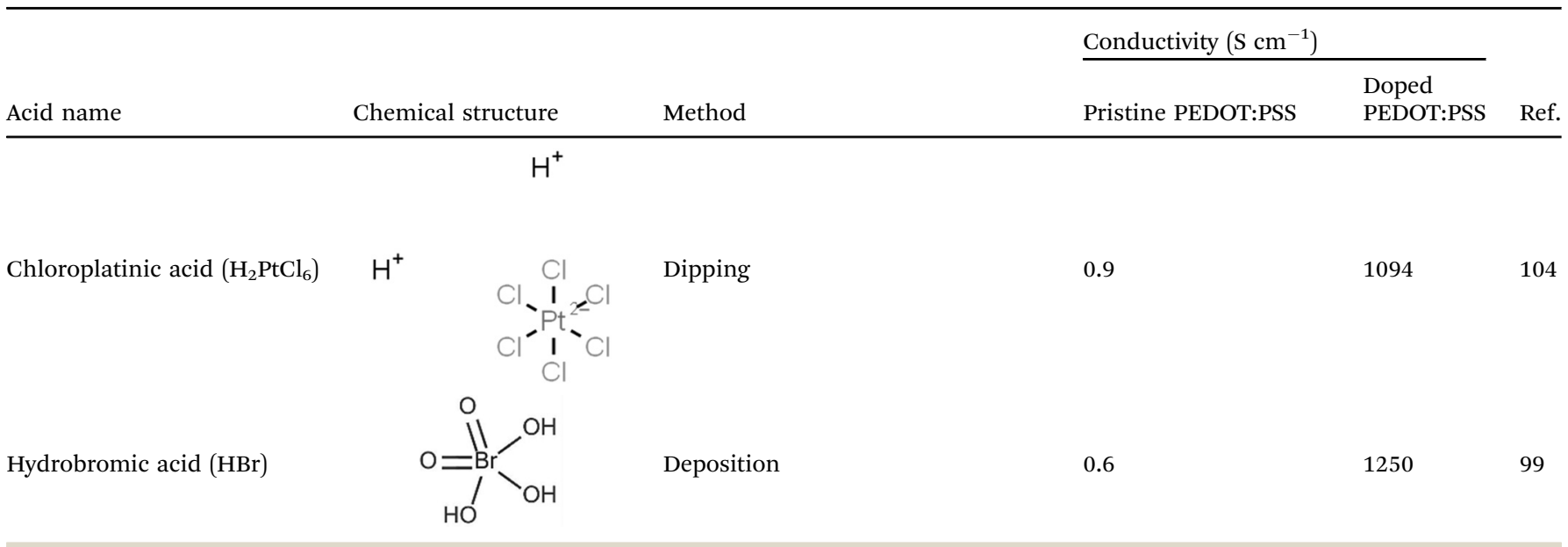

Table 4 Metal nanoparticles incorporated into PEDOT:PSS as reported in the literature

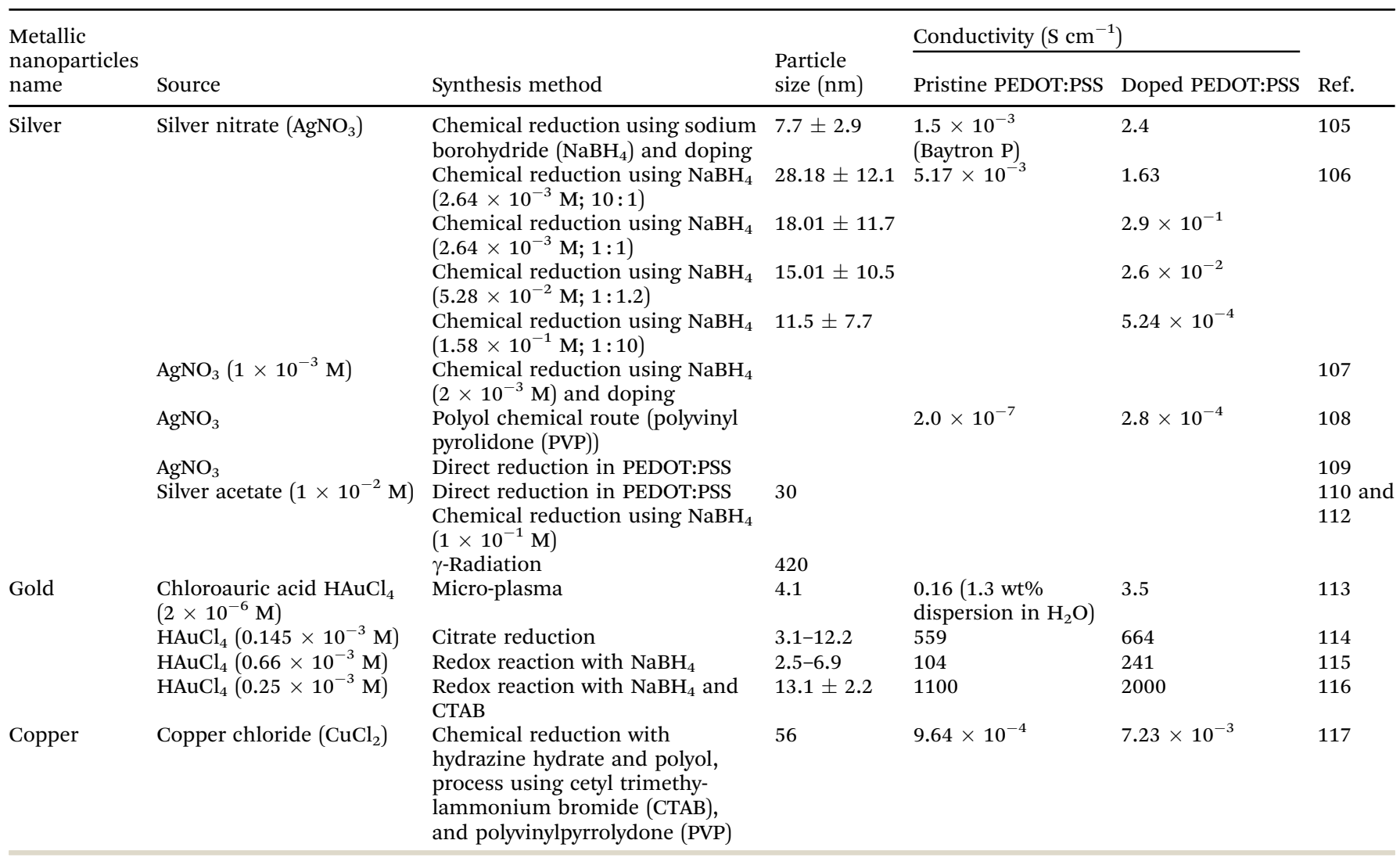

nanoparticles. The excess PSS gives the PEDOT:PSS complex an anionic nature that further promotes the consistency of the silver nanoparticles. The synthesized silver nanoparticles that were functionalized in situ with PEDOT:PSS were demonstrated. ${ }^{105}$ The electrical conductivity of PEDOT:PSS-doped silver nanoparticles (Ag NPs) increased by three orders of magnitude. Subsequently, the influence of Ag NPs synthesized by different molar ratios of the reducing reagent (i.e. $\mathrm{NaBH}_{4}$ ) was investigated. It was found that the electrical conductivity $(\sigma)$ improved as the size of the silver nanoparticles increased, reaching nearly $1.63 \mathrm{~S} \mathrm{~cm}^{-1}$ from $5.17 \times 10^{-3} \mathrm{~S} \mathrm{~cm}^{-1}$. The results were verified by the schematic representation in Fig. $3,{ }^{106}$ where large silver nanoparticles could possibly encounter more than one PEDOT islands simultaneously, thus bridging the distance between different PEDOT islands in PSS isolation. Greater surface interaction occurred between PEDOT and the bridging silver 


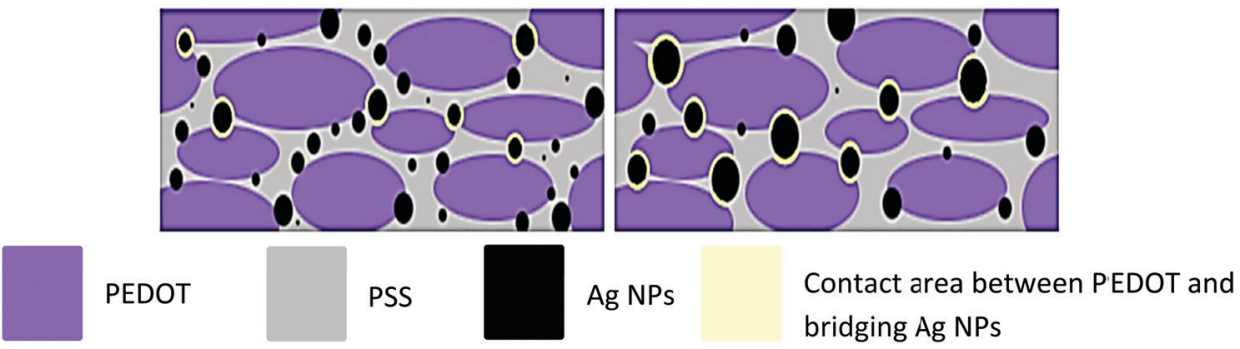

Fig. 3 Schematic illustration of the structure of the films formed by the ink-jet printing of silver nanoparticles doped-PEDOT:PSS. Adapted with permission, ${ }^{111}$ Copyright $(C) 2013$, Elsevier.

nanoparticles (yellow area). Since the functions of silver and PEDOT:PSS are similar, more efficient conducting networks can be combined with larger silver nanoparticles, resulting in higher thin-film efficiency. In this case, the Ag NPs acted as the encapsulating agent for the conducting polymer, enabling electrical performance improvement by forming a combination between the metal core and a conductor polymer shell. The electrical conductivity pattern showed that larger particles lead to higher conductivity. Two conceptual mechanisms can potentially describe the result. First, the formation of silver nanoparticles disrupted the complexation of PEDOT with PSS, prompting the PEDOT chains to form a more extensive conformation related to a more conductive form of quinoid. Second, PSS is an insulating material that blocks the flow of charge carriers through the conductive PEDOT chains. The interaction between $\mathrm{PSS}^{-}$and $\mathrm{Ag}^{+}$could be established to solve this problem, in which the silver nanoparticles serve as electrical connections between the isolating PSS chains and the conductive PEDOT chains. In addition, the existence of Ag NPs on the PEDOT:PSS has been claimed to offer the least resistance path to electrons, thus allowing quick electron movement through the PEDOT:PSS/Ag film, suitable for supercapacitance application. ${ }^{105}$ The Ag NPs embedded with PEDOT:PSS showed increased conductivity ranging from $19 \%$ to $85 \%$, thus offering great potential as a layer in the solar cell application. ${ }^{110}$ Therefore, Ag NPs offer promising characteristics to the PEDOT:PSS for several applications by increasing its conductivity by up to two orders of magnitude. Even though the synthesis of Ag NPs is time consuming and there were errors during the process, it is expected to give a long-life end-product such as sensors and solar cells, resulting in worthwhile experiences for users.

Gold nanoparticles (Au NPs) have been extensively explored for biomedical applications in drug delivery, cancer treatments, and imaging, as well as for biological and chemical sensing, catalysis, and solar cells. ${ }^{114}$ The synthesis of Au NPs to yield $\mathrm{Au}^{0}$ atoms from the reduction reaction of an aqueous solution comprising gold salt pre-cursors like chloroauric acid $\left(\mathrm{HAuCl}_{4}\right)$ is suggested. ${ }^{114-116}$ TSH and DT thiols were used as the capping reagents for $\mathrm{Au}$ NPs. Thiol-capped $\mathrm{Au}$ NPs were created by reducing $\mathrm{HAuCl}_{4}$ with $\mathrm{NaBH}_{4}$ in the presence of the appropriate thiol which resulting in the particle size of around $2.5-2.8 \mathrm{~nm}$ for $\mathrm{Au}-\mathrm{TSH}$ NPs and $6.9 \mathrm{~nm}$ for Au-DT NPs. ${ }^{115}$ PEG-thiol has also been investigated as a protective ligand for $\mathrm{Au}$ NPs; the average diameter of the nanoparticles was about $13.1 \mathrm{~nm} .{ }^{116}$ Capping agents are necessary to stabilize the extracted Au NPs before doping with PEDOT:PSS. It was realized that the Au NPS had a larger particle size, thus affecting the conductivity of PEDOT:PSS because of the higher carrier mobility where $\mathrm{Au}$ NPs are in direct contact with PEDOT molecules, enhancing carrier hopping while reducing carrier concentration. ${ }^{114,115}$ The methods proposed for preparing Au NPs to incorporate with PEDOT:PSS opens up wide opportunities for developing electrodes to serve specific purposes in industry.

The advantage of copper nanoparticles (Cu NPs) over Ag NPs and $\mathrm{Au}$ NPs is their relatively low cost. ${ }^{117}$ However, Cu NPs are easily oxidized at ambient temperature, making it difficult to employ. Hence, it has been suggested that chemical reduction from $\mathrm{CuCl}_{2}$ be applied, and capping agents like CTAB and PVP be introduced to minimize oxidation and agglomeration. The enhancement of conductivity for PEDOT:PSS is contributed by $\mathrm{Cu}$ NPs as connectors. Although the conductivity of $\mathrm{Cu}$ NPs is lower than that of Ag NPs and Au NPs, the preparation method of $\mathrm{Cu}$ NPs is claimed to be more economical because of the cheap $\mathrm{Cu}$ precursors. Hence, the utilization of these NPs shows great potential as electrodes for many applications in electronic devices or other suitable purposes.

\subsection{Summary}

In summary, there are numerous methods for enhancing the conductivity of PEDOT:PSS by incorporating secondary dopants. The organic solvents increased the conductivity, exceeding $1000 \mathrm{~S} \mathrm{~cm}^{-1}$, and it was then further improved to more than $2000 \mathrm{~S} \mathrm{~cm}^{-1}$ by the application of ionic liquids. Other compounds such as acids can also enhance the conductivity of PEDOT:PSS to more than $4000 \mathrm{~S} \mathrm{~cm}^{-1}$ post-treatment. Interestingly, the introduction of metal nanoparticles has brought a new concept to the manufacture of PEDOT:PSS as a nanocomposite, thus offering promising properties for bioelectronic applications.

\section{Conductivity enhancement mechanisms of PEDOT:PSS}

Despite the progress made in enhancing the electrical conductivity of PEDOT:PSS by various methods, whether physical or 
chemical, the mechanism for these conductivity enhancements is debatable. The PEDOT:PSS conductivity enhancement mechanism is assumed to be dependent on the types of chemicals used for treatment, the properties of the chemicals, and the method of treatment applied. Until now, several researchers have suggested various mechanisms..$^{22,33,34,47,51,69,93,105,118-120}$ The most stated view from various research papers is the eradication of slight PSS from PEDOT:PSS, possibly due to the phase separation between PEDOT and PSS, as well as the conformation of PEDOT chains from a coil structure to an extended coil or linear structure. Based on the previous section, PEDOT:PSS is a polyelectrolyte consisting of the closely bound, positively charged conjugated PEDOT and the negatively charged non-conjugated PSS due to strong coulombic attractions. In the pristine film, PEDOT and PSS create a core-shell structure where the core region is rich in conductive PEDOT and the shell region is rich in insulating PSS. SInce the conductive centre is surrounded by the insulating layer, it is difficult for the carriers on the PEDOT chain to pass through the film, resulting in the low conductivity of the pristine film. Therefore, secondary doping is necessary to improve the conductivity of PEDOT:PSS.

\subsection{Screening effect in PEDOT:PSS by solvents}

The screening effect is as important as the polar solvent, which reduces the coulombic interaction between PEDOT $^{+}$and PSS $^{-}$ chains to improve conductivity, though typically the PEDOT: PSS solution is more stable before organic solvents are added. Researchers have focused on the methods of treating PEDOT: PSS films with polar organic compounds for this purpose. As such, the conductivity of PEDOT:PSS has been improved by adding tetrahydrofuran (THF), dimethylformamide (DMF), or dimethyl sulfoxide (DMSO) solvents, mainly due to the polar solvent screening effect. ${ }^{62}$ It was observed that on adding DMSO, as compared to DMF and THF, the PEDOT:PSS's conductivity was boosted by up to two orders of magnitude at room temperature. Due to the high dielectric constant of added polar solvents, the screening effect was assumed to occur between the charge carriers and counter-ions. This lowers the coulombic interaction between PEDOT and PSS, thereby improving the PEDOT:PSS conductivity. ${ }^{36}$

The addition of sorbitol to PEDOT:PSS, on the other hand, allows the ordering of polymer chains during film formation, and the driving force for increased conductivity is the interaction of the dipoles in the polar solvent with the positive charges of PEDOT. ${ }^{70}$ As such, the interaction of sorbitolPEDOT:PSS via hydrogen bonding leads to a screening effect. Additionally, sorbitol treatment screens the ionic interaction between PEDOT and PSS to improve ionic bonding. ${ }^{18}$ The screening effect leads to a successful phase-separation between PEDOT and PSS. This re-orients the coiled shape of PEDOT and PSS chains as shown in Fig. 4a. Besides, PEG/EG treatment screens the ionic interaction between PEDOT and PSS by forming hydrogen bonds with both PSS $^{-}$and PSSH. ${ }^{53}$ This results in a strong step separation between PEDOT and PSS, allowing the linear orientation of the PEDOT chains, as shown in Fig. $4 \mathrm{~b}$.

The polar structure of acidic ionic liquids may be one reason for the higher conductivity. There is the tendency for acidic ionic liquids to remain between PEDOT and PSS, to cause further distance between the two forms, and to prevent chains tangling around themselves. Due to the ionic liquid behavior, it allows direct electron transport so that electron transport from one chain to another can be easily accomplished and the hopping behavior can be improved. Therefore, ionic liquids transform the twisted chains into a linear mode, providing a lower electron transmission path and drawing on the core/shell structure to boost conductivity through the hopping loop. ${ }^{98}$

\subsection{Phase segregation in PEDOT:PSS}

Another mechanism for the conductivity enhancement of PEDOT:PSS is the division of phases. The phase segregation between PEDOT and PSS was revealed on the surfaces by adding secondary dopants such as DMF, EG, sorbitol, and DMSO to produce PEDOT:PSS film. This activity enhanced the electrical conductivity of PEDOT:PSS from $0.5 \mathrm{~S} \mathrm{~cm}^{-1}$ to $775 \mathrm{~S} \mathrm{~cm}^{-1}$. A gradual increase in secondary dopant concentration resulted in the separation of PSS-rich islands from the conductive network of PEDOT resulting in a better direction of conductivity and increased electrical conductivity. The observed phase separation between PEDOT and PSS was assigned to the secondary doping mechanism and is not contingent on the secondary doping content, as evidenced by the phase shift probability profiles. ${ }^{121}$

Ionic liquids, such as 2-methylimidazolium hydrogen sulfate $\left(\mathrm{C}_{4} \mathrm{H}_{8} \mathrm{~N}_{2} \mathrm{O}_{4} \mathrm{~S}\right)$, have a beneficial relationship with both PEDOT and PSS chains. Due to these interactions, PEDOT chains use
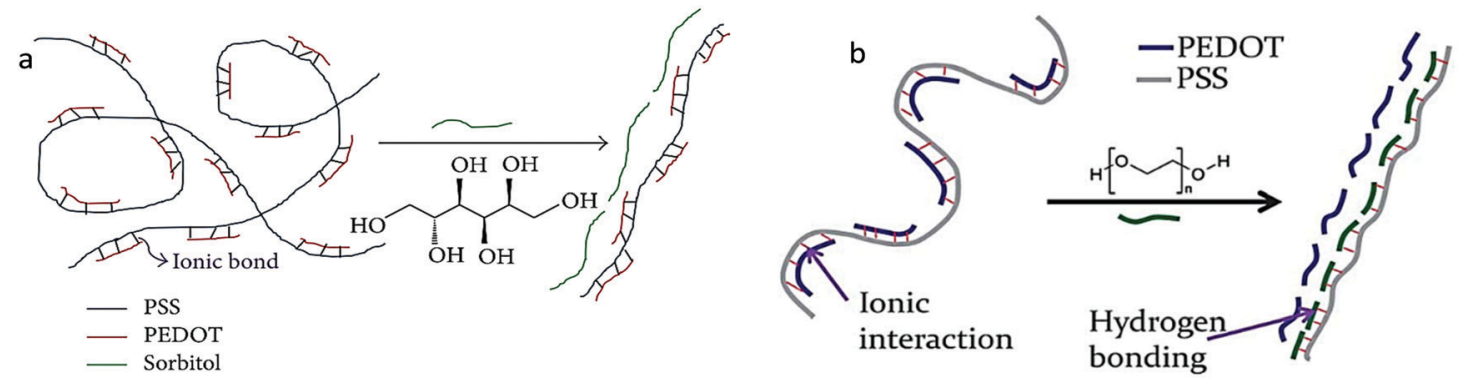

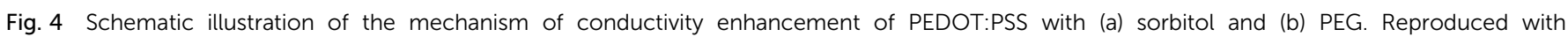
permission, ${ }^{18}$ Copyright (C)2013, Hindawi. ${ }^{51}$ Copyright $(C) 2013$, Royal Society of Chemistry. 

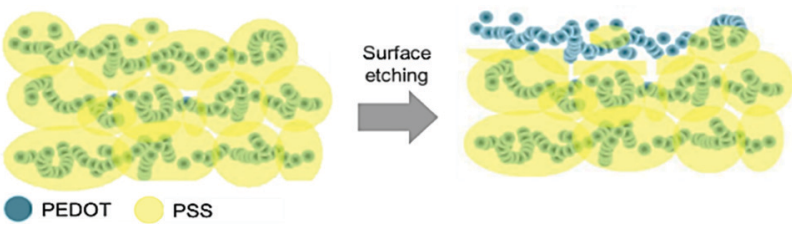

Fig. 5 Conceptual diagram of the cross-sectional view of the PEDOT:PSS film undergoing phase separation. Adapted with permission, ${ }^{124}$ Copyright $(\mathbb{C} 2018$, John Wiley and Sons.

$\mathrm{C}_{4} \mathrm{H}_{8} \mathrm{~N}_{2} \mathrm{O}_{4} \mathrm{~S}$ to distinguish the chains from PSS. This resulted in decreased coulombic interactions between PEDOT and PSS. The hydrophobic PEDOT and hydrophilic PSS chains were separated from each other in phase. Similar findings were also reported in which depletion of the insulating PSS induced a highly conductive PEDOT 3D conducting network and resulted in an improvement in PEDOT:PSS conductivity treated with both EG and PEG. The phase image obtained by AFM showed that the pristine films were homogeneous with disconnected PEDOT chains and weak phase separation between PEDOT and PSS, while there was a good phase separation between PEDOT chains and PSS chains with more fiber-like, conductive PEDOT chains after films. ${ }^{98}$ Fig. 5 illustrates the behavior of PEDOT during the phase segregation process from PSS, leading to increased electrical conductivity.

\subsection{Conformational modifications in the PEDOT:PSS structure}

Conformational modifications to the PEDOT:PSS structure have also led to the enhancement of conductivity. Previous researchers reported that Atomic Force Microscopy (AFM) revealed a twisted wire-like structure that was transformed after the post-treatment with DMSO from a core-shell structure. This was due to the reduction in coulombic interaction between PEDOT and PSS, which resulted in the phase-by-phase separation of the hydrophobic PEDOT from the hydrophilic PSS. ${ }^{27}$ Simultaneously, the presence of the S(2p) peak in the XPS spectra suggested that the decline in coulombic interactions between PEDOT and PSS may be due to the screening effect between the PEDOT $^{+}$chains and PSS $^{-}$chains. ${ }^{51}$ While also eliminating PSS, PEDOT chains changed from a coiled structure to a linear form. This conformation change helped to reduce the energy barrier of charge hopping on the PEDOT:PSS film that also resulted in conductivity improvement. The findings were further confirmed by Raman spectroscopy, with the presence of the strongest band between $1400 \mathrm{~cm}^{-1}$ and $1500 \mathrm{~cm}^{-1}$. The band referred to the $C_{-} \alpha=C_{-} \beta$ symmetrical stretching by the five constituent thiophene rings on the PEDOT chains. Raman spectroscopy provided some details about the polymer chain's intramolecular conductivity, which were verified by electronic absorption spectra. This indicates that the resonant structure of the PEDOT chains differed from the benzenoid structure, which would also be preferred for coil conformation to the quinoid structure, and preferred for the extended coil or linear conformation as shown in Fig. $6 .^{51,93,123}$ The reorientation of the PEDOT polymer chains from coiled to linear or extended-coil configuration allows for even greater interchain contact between the conductive polymers. The energy barrier for hopping inter-chain and inter-domain charges will therefore be reduced, leading to a good transfer of charges between PEDOT chains. Additionally, the linear structure of PEDOT-rich chains, as well as greater grain size, and weaker inter-grain hopping facilitate charge hopping, thereby contributing to higher conductivity. PEDOT:PSS films fabricated by adding secondary dopants such as DMSO, EG, DMF, or sorbitol have shown conductivity enhancement based on the previous statement. ${ }^{24} \mathrm{~A}$ comparable result has also been reported for ionic liquids. ${ }^{98}$ In a PEDOT:PSS film, both coil and linear or extending-coil conformations appear, but once ionic liquids that minimize coulombic interaction are added, the coil conformation becomes a linear or extendingcoil conformation that boosts conductivity. This alteration of the conformation increases the free volume and enhances chain interaction to promote conductivity based on the hopping mechanism.

Similar findings of surface topography and phase separation in PEDOT:PSS-doped EG films were observed via AFM by presenting good uniformity, thus giving the PEDOT-rich grains an up-close connection and integrated phase separation, that further resulted in increased conductivity. These findings suggest that solvents were added to rearrange the conductive PEDOT particles and to isolate the PSS washed off from the surface, thus providing a better route for charge movement in the film, resulting in increased conductivity. a

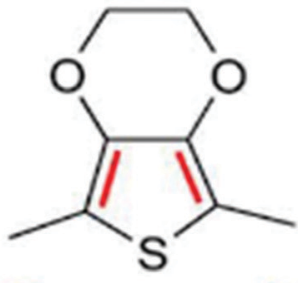

Benzenoid

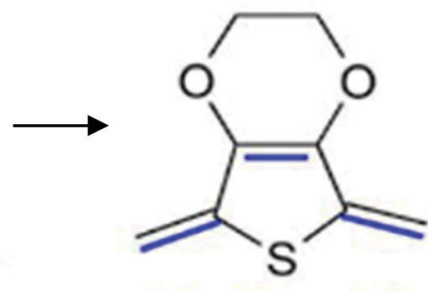

Quinoid b

(i)

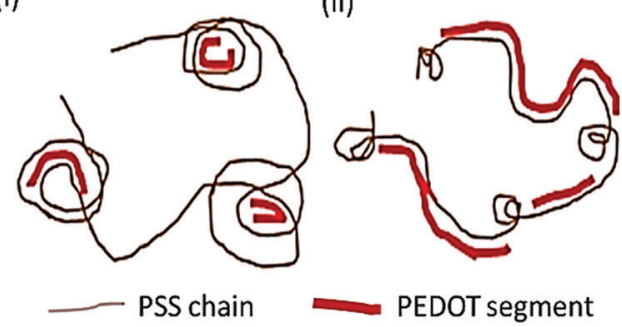

Fig. 6 Schematic structure of the conformational change (a) from benzenoid to quinoid depicted by the blue and red colour in the sketch of the PEDOT molecular structure, and (b) the proposed structure of PEDOT:PSS. Adapted with permission, ${ }^{33}$ Copyright (C) 2015, John Wiley and Sons. ${ }^{97}$ Copyright $(2019$, MDPI. 
a

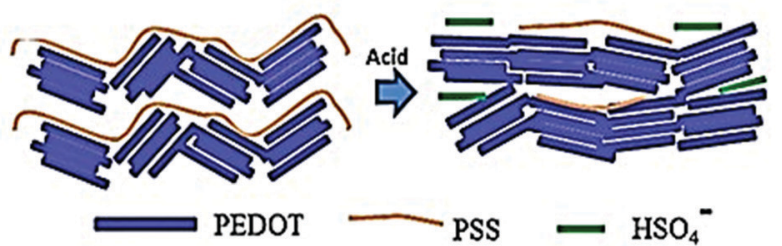

b

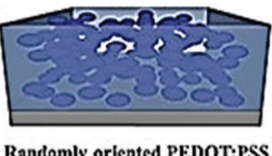

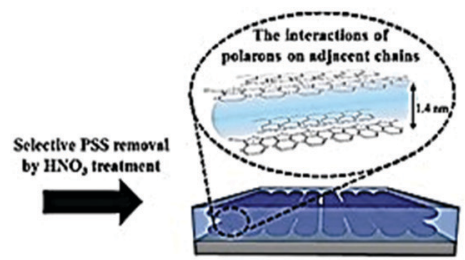

Well ordered and aligned PEDOT:PSS

Fig. 7 Schematic representation of the structural reorientation of the PEDOT:PSS films by (a) $\mathrm{H}_{2} \mathrm{SO}_{4}$ and (b) $\mathrm{HNO}_{3}$ treatment. Adapted with permission, ${ }^{121}$ Copyright (C)2015, Royal Society of Chemistry. ${ }^{102}$ Copyright $(\mathrm{C} 2015$, John Wiley and Sons.

In the case of acid treatment, it is important to note that the $\mathrm{H}^{+}$from the acid neutralizes the $\mathrm{R}-\mathrm{SO}_{3}{ }^{-}$ion group from PSS during the preparation of PEDOT:PSS with strong acid treatment to enhance the conductivity, causing a weakening of the coulombic attraction between PEDOT and PSS. As seen in the equation below, there was phase separation between PSSH chains and the PEDOT:PSS matrix.

$$
\mathrm{H}^{+}+\mathrm{PSS}^{-} \rightarrow \mathrm{PSSH}
$$

The chains are easily washed by deionized water from the PEDOT:PSS matrix, leaving the crystalline PEDOT, which tends to exhibit high conductivity. At the same time, PEDOT chains formed a more oriented structure. ${ }^{123}$ Fig. $7 \mathrm{a}$ and b portray the structural rearrangement or reorientation of PEDOT:PSS films by the modification with $\mathrm{H}_{2} \mathrm{SO}_{4}$ and $\mathrm{HNO}_{3}$.

\subsection{The inherent molecular structure of PEDOT:PSS}

Another mechanism for the conductivity enhancement of PEDOT:PSS is by the inhibited structure itself., ${ }^{3,122}$ A material's electrical conductivity is essentially determined by its electronic structure. The theory of energy bands is a helpful tool for understanding the variations between insulators, semiconductors, and conductors. Theoretically, the band gap is the difference in energy between a material's valence and conduction bands. For insulators, the gap in the band is too wide to be filled by electrons, so they do not conduct electricity. Semiconductors have a narrow energy gap that can be crossed by electrons when excited to enter the conduction band, creating a hole apart. This enables the transportation of both hole and electron charges, which causes current to be conducted. In the case of conductors, when the valence band coincides with the conduction band, the valence electrons can pass and spread in the conduction band, which is an inherent attribute of the conductors.

However, the energy band theory does not explicitly explain how CPs conduct electricity as organic materials. Several researchers have dealt with the molecular-level transport properties of CPs. The widely accepted electronic characteristics of pure CPs from the chemists' perspective is the existence of single- and double-conjugated bonds across the polymer skeleton. Both single and double bonds comprise a localized $\sigma$-bond, creating a tight chemical bond. Conversely, every double bond even comprises a weaker, localized $\pi$-bond. The $\pi$-bond between the first and second atoms of carbon is transported to the spot between the second and the third atoms. In exchange, the $\pi$-bond between the third and fourth carbon passes to the next carbon, and so on. Consequently, the electrons in the double bonds pass along the carbon chain, where the $\mathrm{p}_{z}$-orbitals in the $\pi$-bond chain constantly coincide, and the electrons in the $\pi$-bonds pass across the carbon skeleton. Conjugated double bonds thereby further facilitate electrical discharge, but do not render highly conductive polymeric materials.

\subsection{Doping effects of solvent in PEDOT:PSS}

Doping introduces charge carriers (positive or negative) called polarons and bipolarons into the polymers and renders conductivity through p-type doping (oxidation and positively charged polymers) and n-type doping (reduction and negatively charged polymer). ${ }^{3,47,62,120,123}$ These charge carriers are scattered throughout the polymer chains, rendering better electrical conductivity. Dopants may contribute to the PEDOT:PSS chain by withdrawing electrons and transferring the electrons towards the polymer backbone.

A basic interpretation of the doping effect would be that electrons are either removed from the valence band's highest occupied molecular orbital (HOMO) (oxidation) or moved to the conduction band's lowest unoccupied molecular orbital (LUMO) (reduction). This cycle of oxidation/reduction produces charge carriers in the form of polarons (radical ions), bipolarons (dianions or dications), or solitons in the polymer. ${ }^{3,16}$ Specifically, CPs can be divided between degenerate and nondegenerate models, depending on their ground-level bond structures. Degenerate polymers have two similar dimensional structures at ground level, while non-degenerate polymers show two distinct structures at ground level with varying energies, such as benzenoid and quinoid structures in which the benzenoid energy is weaker than the quinoid.

A major improvement in the conductivity of PEDOT:PSS films with an increase in the doping standard by adding dimethyl sulfate has been proven. ${ }^{47}$ The $\mathrm{SO}_{3}{ }^{-}$ions in PSS are known to carry only one negative charge, which is more likely to produce polaronic states, whereas the $\mathrm{SO}_{4}{ }^{2-}$ ions are doubly charged, increasing the probability of creating bipolaron carriers in PEDOT chains. Consequently, the partial replacement of $\mathrm{PSS}^{-}$by $\mathrm{SO}_{4}{ }^{2-}$ ions increases the bipolar output with an ionexchange loop provided by the rise in the doping rate, resulting in improved conductivity. 


\subsection{Fundamental conductivity consideration}

In addition to the conductivity improvement mechanisms for PEDOT:PSS discussed in the preceding sections, the fundamentals should also be considered. Conductivity, denoted by $\sigma$, is calculated from data on surface resistance $\left(R_{\mathrm{sq}}\right)$, as identified by resistivity $(\rho)$ :

$$
\sigma=\frac{1}{\rho}
$$

Concerning the following equation, the surface resistance $\left(R_{\mathrm{sq}}\right)$ and film thickness $(t)$ can also be derived from the electrical conductivity $(\sigma)$ :

$$
\sigma=\frac{1}{R_{\mathrm{sq}} \times t}
$$

In general, the conducting polymer's electrical conductivity $(\mathrm{s})$ is related to the carrier concentration $(n)$ and Hall mobility $\left(\mu_{\mathrm{H}}\right) .^{38}$ The equation is given as follows:

$$
\sigma=e \times \mu_{\mathrm{H}} \times n
$$

$e$ is recognized as electric charging.

These fundamentals are very useful for researchers who investigate the electrical performance of any conductive material. The characterization techniques carried out by the researchers would have used these equations in obtaining results. Therefore, these fundamentals play an important role in demonstrating the conductivity of the materials and can be related to the other mechanisms discussed in the previous sections.

\subsection{Temperature-dependence of the conductivity of PEDOT:PSS}

Temperature also affects the flow of electrical conductivity in conducting polymers. Charge hopping among polymer chains through almost every conductive polymer is assumed to be the decisive conduction mechanism. The expanded interchain contact between the conductive PEDOT chains would promote charge hopping. This is articulated through the PEDOT:PSS film conductivity quantification from $295 \mathrm{~K}$ to liquid nitrogen temperature. The conductivity based on the temperature dependence of PEDOT:PSS is as follows:

$$
\sigma(T)=\sigma_{0} \exp \left[-\left(\frac{T_{0}}{T}\right)^{\frac{1}{2}}\right]
$$

$\sigma_{0}$ is the conductivity at infinite temperature and $T_{0}$ is the characteristic temperature. Specifically, $T_{0}=16 /\left(k_{\mathrm{B}} N\left(E_{\mathrm{F}}\right) L_{\|} L_{\perp}{ }^{2}\right)$ is the energy barrier between localized states, $N\left(E_{\mathrm{F}}\right)$ is the density of the states at the Fermi level, and $L_{\|}\left(L_{\perp}\right)$ is the localization length in the parallel (perpendicular) direction. For instance, the conductivity of PEDOT:PSS by adding EG as a secondary dopant was enhanced significantly with decreasing $T_{0} .{ }^{95}$ This reaction shows a relatively low energy barrier between the PEDOT chains and a longer charging localization length. It is thus aligned with an expanded interchain contact and the expected alteration of the structure. The Mott variable range hopping (VRH) framework assigns this action in which the temperature-dependent conductivity obeys the following:

$$
\sigma(T)=\sigma_{0} \exp \left[-\left(\frac{T_{0}^{\alpha}}{T}\right)\right]
$$

Here, $\alpha$ is the power coefficient and represents $1 /(1+D)$, in which $D$ is the function dimensionality in the model of the VRH theory. In other words, the power coefficient $\alpha=1 / 2$ accounts for the transport of $1 \mathrm{D}$ charge, while $\alpha=1 / 4$ signifies the hopping of charge throughout all three dimensions. The authenticity of the PEDOT:PSS's VRH model has been highlighted. ${ }^{24,70,95,103,125}$ The temperature-related conductivity showed 1D VRH charge transport for undoped PEDOT:PSS, while 3D VRH charge transport was seen for highly conductive doped PEDOT:PSS films. ${ }^{33}$

A study regarding conductivity enhancement for PEDOT:PSS incorporated with various concentrations of sorbitol has been proven to fit the temperature-dependence theory, as revealed in Fig. 8a. Based on the figure, a slight increase in conductivity with a linear two-step variation was observed in the range of 20 to $80{ }^{\circ} \mathrm{C}$, while a further increment in temperature to $120{ }^{\circ} \mathrm{C}$ showed a rapid increase in conductivity. In addition, the relationship between the weaker energy barrier with the increment of the electrical conductivity of PEDOT:PSS films after a solution treatment is due to the reduction of the interchain charge hopping. This indicates that the PEDOT:PSS film solution treatments may promote the hopping of charges between the interchains, and is possibly influenced by two factors. ${ }^{41}$ One is the existence of an insulator from PSSH that also interrupts the hopping of charges between the interchains. The conformation of the PEDOT chains is linked to another. Specifically, charge hopping over linear PEDOT chains is much simpler than over the coil conforming structure of PEDOT chains. The resistivity temperature dependence in PEDOT:PSS can also be fitted with the mechanism of the one-dimensional variable range hopping $(\mathrm{VRH}):^{24}$

$$
R(T)=R_{0} \exp \left[\left(\frac{T_{0}}{T}\right)^{\frac{1}{2}}\right]
$$

$R_{0}$ is the infinite temperature resistivity and $T_{0}$ is perceived as the temperature when distortion triggers the significant separation of energy that arises within localized states. In the case of PEDOT:PSS acid treatment, specifically using $\mathrm{H}_{2} \mathrm{PtCl}_{6}$, the resultant film acts as either a metal or semi-metal at temperatures greater than $230 \mathrm{~K}$, due to a decrease in resistance, as illustrated in Fig. 8b. Reducing the $T_{0}$ value by adding $\mathrm{H}_{2} \mathrm{PtCl}_{6}$ indicates how the conformation change in the PEDOT chains decreased the transportation of charges to pass the energy barrier. To put things differently, charge hopping over linear PEDOT chains becomes much more convenient than with coil conforming PEDOT chains, as mentioned earlier.

\subsection{Electrochemical behaviour of PEDOT:PSS}

The electrochemical behaviour is essential in the field of bioelectronics, which is associated with a key element called 
a

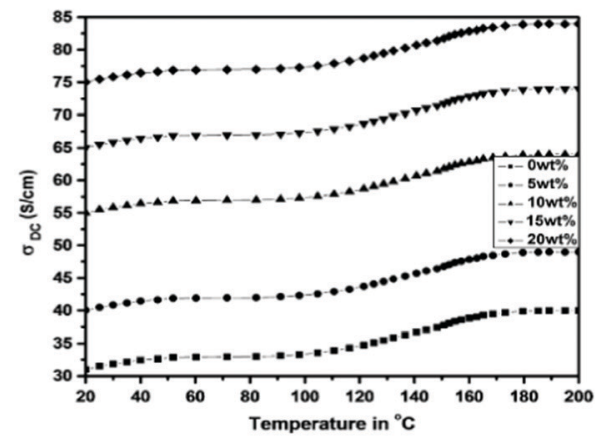

b

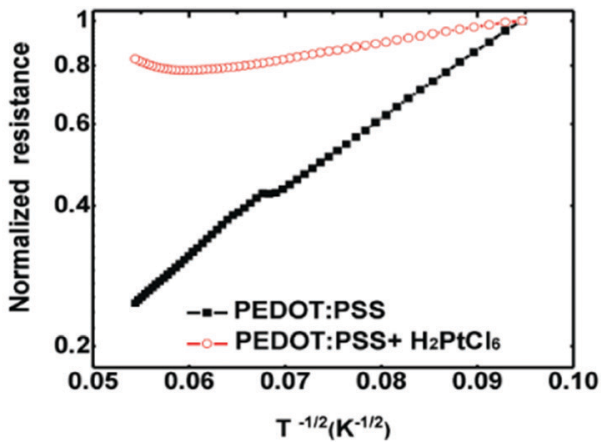

Fig. 8 Temperature dependence of the (a) conductivity and (b) resistivity of undoped and doped PEDOT:PSS. Adapted with permission, ${ }^{103}$ Copyright (C2017, John Wiley and Sons.

the organic electrochemical transistor (OECT) ${ }^{42}$ The OECT often operates with a p-type conducting polymer; PEDOT:PSS is the most commonly used. Generally, the electrochemical behaviour of pristine PEDOT:PSS follows the electrochemical reactions below: ${ }^{109}$

$$
\text { PEDOT }^{+} \mathrm{PSS}^{-}+\mathrm{H}^{+}+\mathrm{e}^{-} \leftrightarrow \text { PEDOT }^{0}+\mathrm{PSS}^{-} \mathrm{H}^{+}
$$

The redox reactions can be investigated by using cyclic voltammetry (CV). CV is an electrochemical method that uses a set scan rate in $\mathrm{mV} \mathrm{s}^{-1}$ to measure the current generated by an applied voltage. $\mathrm{CV}$ is required to analyse redox processes in conductive polymers (CPs), where reduction results in negatively charged polymer chains and oxidation results in positively charged polymer chains during redox reactions. This charge and discharge are related to the doping and de-doping of the CPs. As a result, ions migrate into and out of the polymer matrix during doping and de-doping. After that, given a reversible mechanism, the current peak is computed by the following: ${ }^{3}$

$$
i=n^{2} F^{2} A T v\left(\frac{\exp \theta}{R T(1+\exp \theta)}\right)
$$

where $n$ is the electron number, $F$ is the surface coverage, $A$ is the area of the electrode in $\mathrm{cm}^{2}, \nu$ is the potential scan rate, and $\theta=(n F / R T)\left(E-E^{\circ}\right)$. It is noted that the equation is only valid for low diffusion coefficient dopant ions in thin films of CPs. The occurrence of the mechanism has been examined in experiments on the electrochemical behaviour of PEDOT:PSS incorporated with silver nanoparticles (Ag NPs) as the electrode for electrochemical supercapacitors. ${ }^{109}$ In these circumstances, the cyclic voltammograms show balanced redox peaks with one-electron redox processes ranging from -0.3 and $+1.0 \mathrm{~V}$. The increasing potential scan rate $(\nu)$ was found to be linearly proportional to the current peak observed in the voltammograms. The findings are associated with the presence of Ag NPs in the PEDOT:PSS matrix, where the Ag NPs electrochemical reactions can be justified as follows:

$$
\mathrm{Ag}_{n}{ }^{0} \leftrightarrow \mathrm{Ag}_{n}{ }^{+}+\mathrm{e}^{-}
$$

Based on the results, the current density of PEDOT:PSS increased from 0.29 to $0.37 \mathrm{~mA} \mathrm{~cm}{ }^{-2}$ after incorporating $\mathrm{Ag}$ NPs. This is associated with the charge hopping of Ag NPs metallic conductors through PEDOT:PSS. The findings were also supported by the studies on the electrochemical behaviour of PEDOT:PSS incorporated with gold nanoparticles (Au NPs), where the current density peak showed enhancement. ${ }^{113}$

One of the challenges in approaching the electrochemical behaviour of PEDOT:PSS is to determine the optimum potential range for achieving the maximum current peak. This is to ensure that the performance of the electrode in electronic devices can be maintained for a long time of usage. Overoxidation, on the other hand, is a situation in which the CPs undergo rapid structural degradation and electroactivity loss. Thus, it is recommended to investigate the maximum potential scan rate of the CPs electrode before development for specific applications.

\subsection{Nanotechnology in PEDOT:PSS}

Nanotechnology has emerged in the advances of CP utilization in applications like bioelectronics. Incorporating nanomaterials into the fabrication of CPs leads to the enhancement of the electronic devices suitable for their applications. Nanomaterials like silver nanowires or nanoparticles, carbon nanotubes, graphene oxide, and nanofibrillar cellulose are often used as secondary dopants for PEDOT:PSS. ${ }^{17}$ The fabrication of PEDOT:PSS films with the incorporation of these dopants is usually a simple and straightforward method, such as drop-casting, spin-coating, inkjet printing, and screen-printing techniques.

Silver nanoparticles (Ag NPs) are widely used to produce conductive inks based on PEDOT:PSS. The ink formulation is necessary nowadays to produce flexible and conductive electrodes for various applications, especially in the biomedical field. ${ }^{126}$ It has been reported that a Ag-grid was inkjet printed in a honeycomb configuration and embedded in PEDOT:PSS electrodes to achieve homogeneous electric fields and charge carrier injection, leading to uniform light emission. ${ }^{127}$ The efficiency of $250 \%$ was improved as compared to the ITO reference device when embedding Ag NPs in PEDOT:PSS via the inkjet printing method. Similarly, the uniform composite film fabrication to ensure the good conductivity of the Ag nanowires-doped PEDOT:PSS was proved by undergoing a screen-printing process for optoelectronics. ${ }^{128}$ The screenprinting technique has also utilized halloysite nanotubes (HNT) 
with PEDOT:PSS specifically for dye-sensitized solar cells (DSSC). ${ }^{129}$ The conductivity increased significantly from $358 \mathrm{~S} \mathrm{~cm}^{-1}$ to $381 \mathrm{~S} \mathrm{~cm}^{-1}$ at a low concentration (1 wt\%) of HNT. It was also pointed out that simply homogenizing PEDOT:PSS/ HNTs high-viscosity inks resulted in printed composite electrodes with superior electrical characteristics by two orders of magnitude as compared to electrodes coated with low-viscosity dispersions (7 wt $\%$ ) generated by the drop-casting method.

The processes involving nanomaterials demonstrate the promising properties of PEDOT:PSS in its improved performance as an electrode and sensor. It is also suitable for largescale development in industry, as well as for wider device applications in the near future.

\subsection{Summary}

The mechanisms for the conductivity enhancement of doped PEDOT:PSS have been discussed thoroughly. The most common mechanisms that have been reported are the phase segregation between PEDOT and PSS, and the conformational change of PEDOT chains from a coil structure to an extended coil or linear structure. In addition, the inherent molecular structure of PEDOT:PSS, which includes the conjugated bonds existing within the polymer chains and doping effects toward the energy band of the PEDOT:PSS would also affect the electrical performance. Moreover, fundamental conductivity, as well as charge hopping based on the temperature-dependence characteristic of the PEDOT:PSS should also be considered for conductivity improvement. Overall, these conductivity enhancement mechanisms for doped PEDOT:PSS are related to each other and could be very helpful to other researchers for further work.

\section{Conclusion and outlook}

The primary advances on the electrical conductivity enhancement of PEDOT:PSS have been comprehensively implemented throughout this review paper. Numerous strategies to potentially boost the conductivity of PEDOT:PSS play a vital role, together with processing techniques like solvent additive, post-treatment, ionic liquid additive, acid treatment, and metal nanoparticle additive. The source and mechanism for improving PEDOT:PSS conductivity have been unclear so far, therefore, in this paper, we clarified those issues. However, the most widely accepted interpretation is phase separation between PEDOT and PSS and conformational improvements through PEDOT:PSS rearrangement chains, as discussed in this paper. Therefore, the mechanisms outlined in this paper are expected to provide additional guidance and insight for further conductivity improvement, resulting in further theoretical implications of doped PEDOT:PSS. Overall, the doped PEDOT:PSS offers wide potential usage in many applications and is promising for the advancement of technology.

\section{Author contributions}

Nur'Aishah Ahmad Shahrim wrote the manuscript and performed the data collection. Zuraida Ahmad provided the conceptualization. All authors reviewed and edited the final manuscript.

\section{Conflicts of interest}

There are no conflicts to declare.

\section{Acknowledgements}

The authors would like to acknowledge the financial support provided by Fundamental Research Grant Scheme, Ministry of Education Malaysia (FRGS19-059-0667, FRGS/1/2018/ TK05/UIAM/02/2) and International Islamic University Malaysia (IIUM).

\section{References}

1 R. Balint, N. J. Cassidy and S. H. Cartmell, Conductive polymers: towards a smart biomaterial for tissue engineering, Acta Biomater., 2014, 10, 2341-2353.

2 G. Kaur, R. Adhikari, P. Cass, M. Bown and P. Gunatillake, Electrically conductive polymers and composites for biomedical applications, RSC Adv., 2015, 5, 37553-37567.

3 T. H. Le, Y. Kim and H. Yoon, Electrical and electrochemical properties of conducting polymers, Polymers, 2017, 9, 150-182.

4 T. Nezakati, A. Seifalian, A. Tan and A. M. Seifalian, Conductive Polymers: Opportunities and Challenges in Biomedical Applications, Chem. Rev., 2018, 118, 6766-6843.

5 B. Guo, L. Glavas and A. C. Albertsson, Biodegradable and electrically conducting polymers for biomedical applications, Prog. Polym. Sci., 2013, 38, 1263-1286.

6 R. Ravichandran, S. Sundarrajan, J. R. Venugopal, S. Mukherjee and S. Ramakrishna, Applications of conducting polymers and their issues in biomedical engineering, J. R. Soc., Interface, 2010, 7, S559-S579.

7 S. Inal, J. Rivnay, A. O. Suiu, G. G. Malliaras and I. McCulloch, Conjugated Polymers in Bioelectronics, Acc. Chem. Res., 2018, 51, 1368-1376.

8 N. K. Guimard, N. Gomez and C. E. Schmidt, Conducting polymers in biomedical engineering, Prog. Polym. Sci., 2007, 32, 876-921.

9 D. Mantione, I. del Agua, A. Sanchez-Sanchez and D. Mecerreyes, Poly(3,4-ethylenedioxythiophene) (PEDOT) derivatives: Innovative conductive polymers for bioelectronics, Polymers, 2017, 9, 354-375.

10 M. F. Zainal and Y. Mohd, Characterization of PEDOT Films for Electrochromic Applications, Polym.-Plast. Technol. Eng., 2015, 54, 276-281.

11 S. Wang, S. Guan, J. Wang, H. Liu, T. Liu, X. Ma and Z. Cui, Fabrication and characterization of conductive poly $(3,4-$ ethylenedioxythiophene) doped with hyaluronic acid/poly (L-lactic acid) composite film for biomedical application, J. Biosci. Bioeng., 2017, 123, 116-125. 
12 P. Sakunpongpitiporn, K. Phasuksom, N. Paradee and A. Sirivat, Facile synthesis of highly conductive PEDOT:PSS: Via surfactant templates, RSC Adv., 2019, 9, 6363-6378.

13 T. O. Magu, A. U. Agobi, L. Hitler and P. M. Dass, A Review on Conducting Polymers-Based Composites for Energy Storage Application, Chem. Rev., 2019, 1, 19-34.

14 N. Joy, G. P. Gopalan, J. Eldho and R. Francis, Conducting Polymers: Biomedical Applications, Biomed. Appl. Polym. Mater. Compos., 2016, 37-89.

15 I. Petsagkourakis, N. Kim, K. Tybrandt, I. Zozoulenko and $\mathrm{X}$. Crispin, Poly(3,4-ethylenedioxythiophene): Chemical Synthesis, Transport Properties, and Thermoelectric Devices, Adv. Electron. Mater., 2019, 5, 1800918.

16 J. J. Lee, S. H. Lee, F. S. Kim, H. H. Choi and J. H. Kim, Simultaneous enhancement of the efficiency and stability of organic solar cells using PEDOT:PSS grafted with a PEGME buffer layer, Org. Electron., 2015, 26, 191-199.

17 D. Valtakari, J. Liu, V. Kumar, C. Xu, M. Toivakka and J. J. Saarinen, Conductivity of PEDOT:PSS on Spin-Coated and Drop Cast Nanofibrillar Cellulose Thin Films, Nanoscale Res. Lett., 2015, 10, 1-10.

18 T. H. Meen, K. L. Chen, Y. H. Chen, W. R. Chen, D. W. Chou, W. H. Lan and C. J. Huang, The effects of dilute sulfuric acid on sheet resistance and transmittance in poly(3,4-thylenedioxythiophene): Poly(styrenesulfonate) films, Int. J. Photoenergy, 2013, 2013, 843410.

19 N. R. Shin, S. H. Choi and J. Y. Kim, Highly conductive PEDOT:PSS electrode films hybridized with gold-nanoparticle-doped-carbon nanotubes, Synth. Met., 2014, 192, 23-28.

20 C. Wang, K. Sun, J. Fu, R. Chen, M. Li, Z. Zang, X. Liu, B. Li, H. Gong and J. Ouyang, Enhancement of Conductivity and Thermoelectric Property of PEDOT:PSS via Acid Doping and Single Post-Treatment for Flexible Power Generator, Adv. Sustainable Syst., 2018, 2, 1800085.

21 D. Pani, A. Dessi, J. F. Saenz-Cogollo, G. Barabino, B. Fraboni and A. Bonfiglio, Fully Textile, PEDOT:PSS Based Electrodes for Wearable ECG Monitoring Systems, IEEE Trans. Biomed. Eng., 2016, 63, 540-549.

22 Y. Xia and J. Ouyang, Significant Different Conductivities of the Two Grades of Poly(3,4-ethylenedioxythiophene): Poly(styrenesulfonate), Clevios P and Clevios PH1000, Arising from Different Molecular Weights, Appl. Mater. interfaces, 2012, 4, 4131-4140.

23 Y. Guo, M. T. Otley, M. Li, X. Zhang, S. Sinha, G. M. Triech and G. A. Sotzing, PEDOT:PSS 'wires' Printed on Textile for Wearable Electronics, ACS Appl. Mater. Interfaces, 2016, 8, 26998-27005.

24 J. Nevrela, M. Micjan, M. Novota, S. Kovacova, M. Pavuk, P. Juhasz, J. Kovac Jr, J. Jakabovic and M. Weis, Secondary doping in poly(3,4-ethylenedioxythiophene):Poly(4- styrenesulfonate) thin films, J. Polym. Sci., Part B: Polym. Phys., 2015, 53, 1139-1146.

25 H. Q. Cui, R. X. Peng, W. Song, J. F. Zhang, J. M. Huang, L. Q. Zhu and Z. Y. Ge, Optimization of Ethylene Glycol Doped PEDOT:PSS Transparent Electrodes for Flexible
Organic Solar Cells by Drop-coating Method, Chin. J. Polym. Sci., 2019, 37, 760-766.

26 W. Meng, R. Ge, Z. Li, J. Tong, T. Liu, Q. Zhao, S. Xiong, F. Jiang, L. Mao and Y. Zhou, Conductivity Enhancement of PEDOT:PSS Films via Phosphoric Acid Treatment for Flexible All-Plastic Solar Cells, ACS Appl. Mater. Interfaces, 2015, 7, 14089-14094.

27 T. R. Chou, S. H. Chen, Y. Te Chiang, Y. T. Lin and C. Y. Chao, Highly Conductive PEDOT:PSS Film by PostTreatment with Dimethyl Sulfoxide for ITO-Free Liquid Crystal Display, Mol. Cryst. Liq. Cryst., 2015, 612, 201-210.

28 S. Zhang, P. Kumar, A. S. Nouas, L. Fontaine, H. Tang and F. Cicoira, Solvent-induced changes in PEDOT:PSS films for organic electrochemical transistors, APL Mater., 2015, 3, 014911.

29 A. K. Jayaram, C. Pitsalidis, E. Tan, C. M. Moysidou, M. F. L. DeVolder, J. S. Kim and R. M. Owens, 3D hybrid scaffolds based on PEDOT: PSS/MWCNT composites, Front. Chem., 2019, 7, 1-9.

30 S. Kim, S. Y. Kim, M. H. Chung, J. Kim and J. H. Kim, A one-step roll-to-roll process of stable AgNW/PEDOT:PSS solution using imidazole as a mild base for highly conductive and transparent films: Optimizations and mechanisms, J. Mater. Chem. C, 2015, 3, 5859-5868.

31 C. Deetuam, D. Weise, C. Samthong, P. Praserthdam, R. R. Baumann and A. Somwangthanaroj, Electrical conductivity enhancement of spin-coated PEDOT:PSS thin film via dipping method in low concentration aqueous DMSO, J. Appl. Polym. Sci., 2015, 132, 1-9.

32 L. Yan, X. Gao, J. P. Thomas, N. Jenner, H. Altounian, K. T. Leung, Y. Meng and Y. Li, Ionically cross-linked PEDOT:PSS as a multi-functional conductive binder for high-performance lithium-sulfur batteries, Sustainable Energy Fuels, 2018, 2, 1574-1581.

33 Q. Li, J. Yang, S. Chen, J. Zou, W. Xie and X. Zeng, Highly Conductive PEDOT:PSS Transparent Hole Transporting Layer with Solvent Treatment for High Performance Silicon/Organic Hybrid Solar Cells, Nanoscale Res. Lett., 2017, 12, 506-514.

34 I. Borazan, A. C. Bedeloğlu and A. Demir, A comparative approach to enhance the electrical performance of PEDOT:PSS as transparent electrode for organic solar cells, Polym. Polym. Compos., 2019, 1-8.

35 J. Saghaei, A. Fallahzadeh and M. H. Yousefi, Improvement of electrical conductivity of PEDOT:PSS films by 2-Methylimidazole post treatment, Org. Electron., 2015, 19, 70-75.

36 L. Wang, F. Jiang, J. Xiong, J. Xu, W. Zhou, C. Liu, H. Shi and Q. Jiang, Effects of second dopants on electrical conductivity and thermopower of poly(3,4-ethylenedioxythiophene):poly(styrenesulfonate)-filled carbon black, Mater. Chem. Phys., 2015, 153, 285-290.

37 M. Yang, Y. Zhang, H. Zhang and Z. Li, Characterization of PEDOT:PSS as a biocompatible conductive material, 2015 IEEE 10th Int. Conf. Nano/Micro Eng. Mol. Syst. NEMS 2015, 2015, 149-151.

38 S. H. Lee, H. Park, S. Kim, W. Son, I. W. Cheong and J. H. Kim, Transparent and flexible organic semiconductor 
nanofilms with enhanced thermoelectric efficiency, J. Mater. Chem. A, 2014, 2, 7288-7294.

39 E. J. Shin, S. S. Han and S. M. Choi, Fabrication of highly electrical synthetic leather with polyurethane/poly(3,4ethylene dioxythiophene)/poly(styrene sulfonate), J. Text. Inst., 2018, 109, 241-247.

40 M. G. Tadesse, D. A. Mengistie, Y. Chen, L. Wang, C. Loghin and V. Nierstrasz, Electrically conductive highly elastic polyamide/lycra fabric treated with PEDOT:PSS and polyurethane, J. Mater. Sci., 2019, 54, 9591-9602.

41 Z. Yu, Y. Xia, D. Du and J. Ouyang, PEDOT:PSS Films with Metallic Conductivity through a Treatment with Common Organic Solutions of Organic Salts and Their Application as a Transparent Electrode of Polymer Solar Cells, ACS Appl. Mater. Interfaces, 2016, 8, 11629-11638.

42 L. V. Lingstedt, M. Ghittorelli, H. Lu, D. A. Koutsouras, T. Marszalek, F. Torricelli, N. I. Crăciun, P. Gkoupidenis and P. W. M. Blom, Effect of DMSO Solvent Treatments on the Performance of PEDOT:PSS Based Organic Electrochemical Transistors, Adv. Electron. Mater., 2019, 5, 1-8.

43 D. Alemu, H. Y. Wei, K. C. Ho and C. W. Chu, Highly conductive PEDOT:PSS electrode by simple film treatment with methanol for ITO-free polymer solar cells, Energy Environ. Sci., 2012, 5, 9662-9671.

44 M. Zahid, E. L. Papadopoulou, A. Athanassiou and I. S. Bayer, "Strain-responsive mercerized conductive cotton fabrics based on PEDOT:PSS/graphene, Mater. Des., 2017, 135, 213-222.

45 T. Horii, H. Hikawa, Y. Mochizuki and H. Okuzaki, Synthesis and Characterization of Highly Conductive PEDOT/ PSS Colloidal Gels, Trans. Mater. Res. Soc. Jpn., 2012, 37, 515-518.

46 U. Lang, N. Naujoks and J. Dual, Mechanical characterization of PEDOT:PSS thin films, Synth. Met., 2009, 159, 473-479.

47 M. Reyes-Reyes, I. Cruz-Cruz and R. López-Sandoval, "Enhancement of the electrical conductivity in PEDOT: PSS films by the addition of dimethyl sulfate, J. Phys. Chem. $C, 2010,114,20220-20224$.

48 S. R. Ha, S. Park, J. T. Oh, D. H. Kim, S. Cho, S. Y. Bae, D. W. Kang, J. M. Kim and H. Choi, Water-resistant PEDOT:PSS hole transport layers by incorporating a photo-crosslinking agent for high-performance perovskite and polymer solar cells, Nanoscale, 2018, 10, 13187-13193.

49 B. Xu, S. A. Gopalan, A. I. Gopalan, N. Muthuchamy, K. P. Lee, J. S. Lee, Y. Jiang, S. W. Lee, S. W. Kim, J. S. Kim, H. M. Jeong, J. B. Kwon, J. H. Bae and S. W. Kang, Functional solid additive modified PEDOT:PSS as an anode buffer layer for enhanced photovoltaic performance and stability in polymer solar cells, Sci. Rep., 2017, 7, 1-12.

50 B. Qu, S. Ma, Z. Chen, L. Xiao and Q. Gong, Indium tin oxide-free polymer solar cells using a PEDOT:PSS/Ag/ PEDOT:PSS multilayer as a transparent anode, J. Phys. D: Appl. Phys., 2012, 45, 425102.

51 D. Alemu Mengistie, P. C. Wang and C. W. Chu, Effect of molecular weight of additives on the conductivity of
PEDOT:PSS and efficiency for ITO-free organic solar cells, J. Mater. Chem. A, 2013, 1, 9907-9915.

52 A. Giuri, S. Masi, S. Colella, A. Listorti, A. Rizzo, A. Liscio, E. Treossi, V. Palermo, G. Gigli, C. Mele and C. E. Corcione, GO/PEDOT:PSS nanocomposites: effect of different dispersing agents on rheological, thermal, wettability and electrochemical properties, Nanotechnology, 2012, 28, 174001.

53 N. Perinka, C. H. Kim, M. Kaplanova and Y. Bonnassieux, Preparation and characterization of thin conductive polymer films on the base of PEDOT:PSS by ink-jet printing, Phys. Procedia, 2013, 44, 120-129.

54 A. Giuri, S. Masi, S. Colella, A. Listorti, A. Rizzo, A. Kovtun, S. Dell'Elce, A. Liscio and C. E. Corcione, Rheological and physical characterization of PEDOT: PSS/graphene oxide nanocomposites for perovskite solar cells, Polym. Eng. Sci., 2017, 57, 546-552.

55 T. Stöcker, A. Köhler and R. Moos, Why does the electrical conductivity in PEDOT:PSS decrease with PSS content? A study combining thermoelectric measurements with impedance spectroscopy, J. Polym. Sci., Part B: Polym. Phys., 2012, 50, 976-983.

56 Z. Zhu, C. Liu, F. Jiang, J. Xu and E. Liu, Effective treatment methods on PEDOT:PSS to enhance its thermoelectric performance, Synth. Met., 2017, 225, 31-40.

57 E. Hrehorova, M. Rebros, A. Pekarovicova, P. D. Fleming and V. N. Bliznyuk, Characterization of conductive polymer inks based on PEDOT:PSS, Proc. Tech. Assoc. Graph. Arts, TAGA, 2007, 4, pp. 244-256.

58 M. Döbbelin, R. Marcilla, M. Salsamendi, C. Pozo-Gonzalo, P. M. Carrasco, J. A. Pomposo and D. Mecerreyes, Influence of ionic liquids on the electrical conductivity and morphology of PEDOT:PSS films, Chem. Mater., 2007, 19, 2147-2149.

59 F. M. Tarmidzi and S. B. Sasongko, Highly Conductive PEDOT: PSS Flexible Film with Secondary Doping and Spray Pyrolysis Method, Int. J. Appl. Eng. Res., 2018, vol. 13, 10234-10239.

60 Z. Hu, J. Zhang and Y. Zhu, Effects of solvent-treated PEDOT: PSS on organic photovoltaic devices, Renewable Energy, 2014, 62, 100-105.

61 Z. Zhu, C. Liu, J. Xu, Q. Jiang, H. Shi and E. Liu, Improving the electrical conductivity of PEDOT:PSS films by binary secondary doping, Electron. Mater. Lett., 2016, 12, 54-58.

62 J. Y. Kim, J. H. Jung, D. E. Lee and J. Joo, Enhancement of electrical conductivity of poly(3, 4-ethylenedioxythiophene)/poly(4-styrenesulfonate) by a change of solvents, Synth. Met., 2002, 126, 311-316.

63 C. Yi, A. Wilhite, L. Zhang, R. Hu, S. S. C. Chuang, J. Zheng and $\mathrm{X}$. Gonget, Enhanced thermoelectric properties of poly(3,4-ethylenedioxythiophene):poly(styrenesulfonate) by binary secondary dopants, ACS Appl. Mater. Interfaces, 2015, 7, 8984-8989.

64 O. P. Dimitriev, D. A. Grinko, Y. V. Noskov, N. A. Ogurtsov and A. A. Pud, PEDOT:PSS films-Effect of organic solvent additives and annealing on the film conductivity, Synth. Met., 2009, 159, 2237-2239. 
65 A. Pasha, A. S. Roy, M. V. Murugendrappa, O. A. Al-Hartomy and S. Khasim, Conductivity and dielectric properties of PEDOT-PSS doped DMSO nano composite thin films, J. Mater. Sci.: Mater. Electron., 2016, 27, 8332-8339.

66 I. Lee, G. W. Kim, M. Yang and T. S. Kim, Simultaneously Enhancing the Cohesion and Electrical Conductivity of PEDOT:PSS Conductive Polymer Films using DMSO Additives, ACS Appl. Mater. Interfaces, 2016, 8, 302-310.

67 H. Yamaguchi, K. Aizawa, Y. Chonan, T. Komiyama, T. Aoyama, E. Sakai, J. Qiu and N. Sato, Highly Flexible and Conductive Glycerol-Doped PEDOT:PSS Films Prepared Under an Electric Field, J. Electron. Mater., 2018, 47, 3370-3375.

68 L. A. A. Pettersson, S. Ghosh and O. Inganäs, Optical anisotropy in thin films of poly(3,4-ethylenedioxythiophene)poly(4-styrenesulfonate), Org. Electron., 2002, 3, 143-148.

$69 \mathrm{~J}$. Li, J. Liu and C. Gao, On the mechanism of conductivity enhancement in PEDOT/PSS film doped with sorbitol, e-Polym., 2011, 11, 1-8.

70 S. Khasim, A. Pasha, A. S. Roy, A. Parveen and N. Badi, Effect of Secondary Doping Using Sorbitol on Structure and Transport Properties of PEDOT-PSS Thin Films, J. Electron. Mater., 2017, 46, 4439-4447.

71 A. M. Nardes, M. Kemerink, M. M. de Kok, E. Vinken, K. Maturova and R. A. J. Janssen, Conductivity, work function, and environmental stability of PEDOT:PSS thin films treated with sorbitol, Org. Electron., 2008, 9, 727-734.

72 N. Kishi, Y. Kondo, H. Kunieda, S. Hibi and Y. Sawada, Enhancement of thermoelectric properties of PEDOT:PSS thin films by addition of anionic surfactants, J. Mater. Sci.: Mater. Electron., 2018, 29, 4030-4034.

73 C. Yeon, G. Kim, J. W. Lim and S. J. Yun, Highly conductive PEDOT:PSS treated by sodium dodecyl sulfate for stretchable fabric heaters, RSC Adv., 2017, 7, 5888-5897.

74 Y. Xia, K. Sun and J. Ouyang, Solution-processed metallic conducting polymer films as transparent electrode of optoelectronic devices, Adv. Mater., 2012, 24, 2436-2440.

75 J. H. Lee, J. H. Lee, Y. R. Jeong, G. Lee, S. W. Jin, Y. H. Lee, S. Y. Hong, H. Park, J. W. Kim, S. S. Lee and J. S. Ha, Highly Conductive, Stretchable, and Transparent PEDOT:PSS Electrodes Fabricated with Triblock Copolymer Additives and Acid Treatment, ACS Appl. Mater. Interfaces, 2018, 10, 1-33.

76 E. Hosseini, V. O. Kollath and K. Karan, The Key Mechanism of Conductivity in PEDOT:PSS Thin Films Exposed by Anomalous Conduction Behaviour upon Solvent-Doping and Sulfuric Acid Post-Treatment, J. Mater. Chem., 2013, 1, 3777.

77 Y. H. Kim, C. Sachse, M. L. MacHala, C. May, L. MüllerMeskamp and K. Leo, Highly conductive PEDOT:PSS electrode with optimized solvent and thermal post-treatment for ITO-free organic solar cells, Adv. Funct. Mater., 2011, 21, 1076-1081.

78 M. N. Gueye, M. N. Gueye, A. Carella, N. Massonnet, E. Yvenou, S. Brenet, J. Faure-Vincent, S. Pouget, F. Rieutord, H. Okuno, A. Benayad, R. Demadrille and
J. P. Simonato, Structure and Dopant Engineering in PEDOT Thin Films: Practical Tools for a Dramatic Conductivity Enhancement, Chem. Mater., 2016, 28, 3462-3468.

79 E. Yildirim, G. Wu, X. Yong, T. L. Tan, Q. Zhu, J. Xu, J. Ouyang, J. S. Wang and S. W. Yang, A theoretical mechanistic study on electrical conductivity enhancement of DMSO treated PEDOT:PSS, J. Mater. Chem. C, 2018, 6, 5122-5131.

80 Y. Xia, K. Sun and J. Ouyang, Highly conductive poly(3,4ethylenedioxythiophene):Poly(styrene sulfonate) films treated with an amphiphilic fluoro compound as the transparent electrode of polymer solar cells, Energy Environ. Sci., 2012, 5, 5325-5332.

81 X. Fan, W. Nie, H. Tsai, N. Wang, H. Huang, Y. Cheng, R. Wen, L. Ma, F. Yan and Y. Xia, PEDOT:PSS for Flexible and Stretchable Electronics: Modifications, Strategies, and Applications, Adv. Sci., 2019, 6, 1900813.

82 N. Chaturvedi and V. Dutta, Effect of electric field on the spray deposited PEDOT: PSS film, Energy Procedia, 2013, 33, 228-232.

83 D. Wang, Y. Mei and G. Huang, Printable inorganic nanomaterials for flexible transparent electrodes: From synthesis to application, J. Semicond., 2018, 39, 011002.

84 M. Sain, S. Ummartyotin, J. Juntaro, C. Wu and H. Manuspiya, Deposition of PEDOT: PSS nanoparticles as a conductive microlayer anode in OLEDs device by desktop inkjet printer, J. Nanomater., 2011, 2011, 606714.

85 A. M. Grancarić, I. Jerkovic, V. Koncar, C. Cochrane, F. M. Kelly, D. Soulat and X. Legrand, Conductive polymers for smart textile applications, J. Ind. Text., 2018, 48, 612-642.

86 Z. Wen, Y. Yang, N. Sun, G. Li, Y. Liu, C. Chen, J. Shi, L. Xie, H. Jiang, D. Bao, Q. Zhuo and X. Sun, A Wrinkled PEDOT:PSS Film Based Stretchable and Transparent Triboelectric Nanogenerator for Wearable Energy Harvesters and Active Motion Sensors, Adv. Funct. Mater., 2018, 28, 1803684.

87 L. V. Kayser and D. J. Lipomi, Stretchable Conductive Polymers and Composites Based on PEDOT and PEDOT:PSS, Adv. Mater., 2019, 31, 1-13.

88 A. De Izarra, S. Park, J. Lee, Y. Lansac and Y. H. Jang, Ionic Liquid Designed for PEDOT:PSS Conductivity Enhancement, J. Am. Chem. Soc., 2018, 140, 5375-5384.

89 Y. Zhou, H. Cheun, S. Choi, W. J. Potscavage, C. FuentesHernandez and B. Kippelen, Indium tin oxide-free and metal-free semitransparent organic solar cells, Appl. Phys. Lett., 2010, 97, 153304.

90 E. Jin Bae, Y. Hun Kang, K. S. Jang and S. Yun Cho, Enhancement of Thermoelectric Properties of PEDOT:PSS and Tellurium-PEDOT:PSS Hybrid Composites by Simple Chemical Treatment, Sci. Rep., 2016, 6, 18805.

91 J. Song, G. Ma, F. Qin, L. Hu, B. Luo, T. Liu, X. Yin, S. Zhen, Z. Zeng, Y. Jiang, G. Wang and Z. Li, High-Conductivity, Flexible and Transparent PEDOT:PSS Electrodes for High Performance Semi-Transparent Supercapacitors, Polymers, 2020, 12, 450 . 
92 Y. J. Lin, W. S. Ni and J. Y. Lee, Effect of incorporation of ethylene glycol into PEDOT:PSS on electron phonon coupling and conductivity, J. Appl. Phys., 2015, 117, 215501.

93 J. Rivnay, S. Inal, B. A. Collins, M. Sessolo, E. Stavrinidou, X. Strakosas and G. G. Malliaras, Structural control of mixed ionic and electronic transport in conducting polymers, Nat. Commun., 2016, 7, 11287.

94 X. Crispin, F. L. E. Jakobsson, A. Crispin, P. C. M. Grim, P. Andersson, A. Volodin, C. van Haesendonck, M. Van der Auweraer, W. R. Salaneck and M. Berggren, The Origin of the High Conductivity of (PEDOT-PSS) Plastic Electrodes, Chem. Mater., 2006, 18, 4354-4360.

95 J. Ouyang, Q. Xu, C. W. Chu, Y. Yang, G. Li and J. Shinar, On the mechanism of conductivity enhancement in poly(3,4- ethylenedioxythiophene):poly(styrene sulfonate) film through solvent treatment, Polymer, 2004, 45, 8443-8450.

96 Y. Li, R. Tanigawa and H. Okuzaki, Soft and flexible PEDOT/PSS films for applications to soft actuators, Smart Mater. Struct., 2014, 23, 074010.

97 S. P. Rwei, Y. H. Lee, J. W. Shiu, R. Sasikumar and U. T. Shyr, Characterization of solvent-treated PEDOT:PSS thin films with enhanced conductivities, Polymers, 2019, 11, 134-144.

98 F. Atabaki, M. H. Yousefi, A. Abdolmaleki and M. Kalvandi, Poly(3,4-ethylenedioxythiophene):Poly(styrenesulfonic Acid) (PEDOT:PSS) Conductivity Enhancement through Addition of Imidazolium-Ionic Liquid Derivatives, Polym.Plast. Technol. Eng., 2015, 54, 1009-1016.

99 R. Bhatia, K. Kumari and R. Gandhi, On the Electrical Conductivity Poly (Styrenesulfonate) Thin Films, Int. J. Nanosci., 2017, 15, 175006.

100 Ö. Yagci, S. S. Yesilkaya, S. A. Yüksel, F. Ongül, N. M. Varal, M. Kus and O. Icelli, Effect of boric acid doped PEDOT:PSS layer on the performance of P3HT:PCBM based organic solar cells, Synth. Met., 2016, 212, 12-18.

101 N. Kim, S. Kee, S. H. Lee, B. H. Lee, Y. H. Kahng, Y.-R. Jo and K. Lee, Transparent Electrodes: Highly Conductive PEDOT:PSS Nanofibrils Induced by Solution-Processed Crystallization, Adv. Mater., 2014, 26, 2109.

102 C. Yeon, S. J. Yun, J. Kim and J. W. Lim, PEDOT:PSS Films with Greatly Enhanced Conductivity via Nitric Acid Treatment at Room Temperature and Their Application as Pt/TCO-Free Counter Electrodes in Dye-Sensitized Solar Cells, Adv. Electron. Mater., 2015, 1, 1-8.

103 F. Wu, P. Li, K. Sun, Y. Zhou, W. Chen, J. Fu, M. Li, S. Lu, D. Wei, X. Tang, Z. Zang, L. Sun, X. Liu and J. Ouyang, Conductivity Enhancement of PEDOT:PSS via Addition of Chloroplatinic Acid and Its Mechanism, Adv. Electron. Mater., 2017, 3, 1700047.

104 E. Amini and M. Azadfallah, In situ synthesis of silver nanoparticles on fiber matrix for preparing antibacterial paper, Biointerface Res. Appl. Chem., 2018, 8, 3449-3456.

105 D. S. Patil, S. A. Pawar, J. Hwang, J. H. Kim, P. S. Patil and J. C. Shin, Silver incorporated PEDOT: PSS for enhanced electrochemical performance, J. Ind. Eng. Chem., 2016, 42, 113-120.
106 S. Xiong, L. Zhang and X. Lu, Conductivities enhancement of poly(3,4-ethylenedioxythiophene)/poly(styrene sulfonate) transparent electrodes with diol additives, Polym. Bull., 2013, 70, 237-247.

107 K. J. Moreno, I. Moggio, E. Arias, I. Llarena, S. E. Moya, R. F. Ziolo and H. Barrientos, Silver nanoparticles functionalized in situ with the conjugated polymer (PEDOT:PSS), J. Nanosci. Nanotechnol., 2009, 9, 3987-3992.

108 R. G. Melendez, K. J. Moreno, I. Moggio, E. Arias, A. Ponce, I. Llanera and S. E. Moya, On the Influence of Silver Nanoparticles Size in the Electrical Conductivity of PEDOT: PSS, Mater. Sci. Forum, 2010, 644, 85-90.

109 D. S. Patil, S. A. Pawar, P. S. Patil, J. H. Kim and J. C. Shin, Silver nanoparticles incorporated PEDOT-PSS electrodes for electrochemical supercapacitor, J. Nanosci. Nanotechnol., 2016, 16, 10625-10629.

110 A. Iwan, B. Boharewicz, I. Tazbir, A. Sikora and B. Zboromirska-Wnukiewicz, Silver Nanoparticles in PEDOT: PSS Layer for Polymer Solar Cell Application, Int. J. Photoenergy, 2015, 2015, 1-9.

111 Z. Xiong, C. Dong, H. Cai, C. Liu and X. Zhang, "Composite inks of poly(3,4-ethylenedioxythiophene)/poly(styrenesulfonate)/silver nanoparticles and electric/optical properties of inkjet-printed thin films, Mater. Chem. Phys., 2013, 141.

112 O. A. Ghazy, M. M. Ibrahim, F. I. A. Elfadl, H. M. Hosni, E. M. Shehata, N. M. Deghiedy and M. R. Balboul, PEDOT: PSS incorporated silver nanoparticles prepared by gamma radiation for the application in organic solar cells, J. Radiat. Res. Appl. Sci., 2015, 8, 166-172.

113 R. C. Zhang, D. Sun, R. Zhang, W. F. Lin, M. MaciasMontero, J. Patel and P. Maguire, Gold nanoparticlepolymer nanocomposites synthesized by room temperature atmospheric pressure plasma and their potential for fuel cell electrocatalytic application, Sci. Rep., 2017, 7, 1-9.

114 N. Toshima and N. Jiravanichanun, Improvement of thermoelectric properties of PEDOT/PSS films by addition of gold nanoparticles: Enhancement of seebeck coefficient, J. Electron. Mater., 2013, 42, 1882-1887.

115 N. Toshima, N. Jiravanichanun and H. Marutani, Organic thermoelectric materials composed of conducting polymers and metal nanoparticles, J. Electron. Mater., 2012, 41, n735-1742.

116 A. Yoshida and N. Toshima, Gold nanoparticle and gold nanorod embedded PEDOT:PSS thin films as organic thermoelectric materials, J. Electron. Mater., 2014, 43, 1492-1497.

117 L. Q. Pham, J. H. Sohn, C. W. Kim, J. H. Park, H. S. Kang, B. C. Lee and Y. S. Kang, Copper nanoparticles incorporated with conducting polymer: Effects of copper concentration and surfactants on the stability and conductivity, J. Colloid Interface Sci., 2012, 365, 103-109.

118 J. Ouyang, 'Secondary doping' methods to significantly enhance the conductivity of PEDOT:PSS for its application as transparent electrode of optoelectronic devices, Displays, 2013, 34, 423-436. 
119 H. Shi, C. Liu, Q. Jiang and J. Xu, Effective Approaches to Improve the Electrical Conductivity of PEDOT:PSS: A Review, Adv. Electron. Mater., 2015, 1, 1-16.

120 Z. Cao, Z. Chen and L. Escoubas, Optical, structural, and electrical properties of PEDOT:PSS thin films doped with silver nanoprisms, Opt. Mater. Express, 2014, 4, 2525.

121 M. Donoval, M. Micjana, M. Novotaa, J. Nevrela, S. Kovacova, M. Pavuk, P. Juhasz, M. Jagelka, J. Kovac Jr, J. Jakabovic, M. Cigan and M. Wei, Relation between secondary doping and phase separation in PEDOT:PSS films, Appl. Surf. Sci., 2017, 395, 86-91.

122 Z. Fan and J. Ouyang, Thermoelectric Properties of PEDOT: PSS, Adv. Electron. Mater., 2019, 1800769, 1-23.

123 S. R. S. Kumar, N. Kurra and H. N. Alshareef, Enhanced high temperature thermoelectric response of sulphuric acid treated conducting polymer thin films, J. Mater. Chem. C, 2015, 4, 215-221.

124 K. Lim, J. Kang, S. Jung, S. Lee, J. Park, D. G. Kim and Y. C. Kang, Improving Electrical Conductivity of PEDOT:PSS with Phase Separation by Applying Electric Fields, Bull. Korean Chem. Soc., 2018, 39, 469-476.
125 T. Horii, H. Hikawa, M. Katsunuma and H. Okuzaki, Synthesis of highly conductive PEDOT:PSS and correlation with hierarchical structure, Polymer, 2018, 140, 33-38.

126 D. Barmpakos, C. Tsamis and G. Kaltsas, Multi-parameter paper sensor fabricated by inkjet-printed silver nanoparticle ink and PEDOT:PSS, Microelectron. Eng., 2020, 225, 111266.

127 L. Kinner, S. Nau, K. Popovic, S. Sax, I. Burgués-Ceballos, F. Hermerschmidt and E. J. W. List-Kratochvil, Inkjetprinted embedded Ag-PEDOT:PSS electrodes with improved light out coupling effects for highly efficient ITO-free blue polymer light emitting diodes, Appl. Phys. Lett., 2017, 110, 101107.

128 X. He, R. He, Q. Lan, W. Wu, F. Duan, J. Xiao and J. Liu, Screen-printed fabrication of PEDOT: PSS/silver nanowire composite films for transparent heaters, Materials, 2017, 10, 220.

129 P. Gemeiner, J. Kuliček, T. Syrový, A. Ház, V. Khunová, M. Hatala and M. Omastová, Screen-printed PEDOT:PSS/ halloysite counter electrodes for dye-sensitized solar cells, Synth. Met., 2019, 256, 116148. 